\title{
DISCONTINUOUS SOLUTIONS OF HAMILTON-JACOBI EQUATIONS VERSUS RADON MEASURE-VALUED SOLUTIONS OF SCALAR CONSERVATION LAWS: DISAPPEARANCE OF SINGULARITIES
}

\author{
MICHIEL BERTSCH, FLAVIA SMARRAZZO, ANDREA TERRACINA, \\ AND ALBERTO TESEI
}

\begin{abstract}
Let $H$ be a bounded and Lipschitz continuous function. We consider discontinuous viscosity solutions of the Hamilton-Jacobi equation $U_{t}+$ $H\left(U_{x}\right)=0$ and signed Radon measure valued entropy solutions of the conservation law $u_{t}+[H(u)]_{x}=0$. After having proved a precise statement of the formal relation $U_{x}=u$, we establish estimates for the (strictly positive!) times at which singularities of the solutions disappear. Here singularities are jump discontinuities in case of the Hamilton-Jacobi equation and signed singular measures in case of the conservation law.
\end{abstract}

\section{IntRoduction}

Let $H$ be bounded and Lipschitz continuous in $\mathbb{R}$, $\left(H_{1}\right)$

$$
H \in W^{1, \infty}(\mathbb{R}),
$$

and consider the Cauchy problem for the first order Hamilton-Jacobi equation

$$
\begin{cases}U_{t}+H\left(U_{x}\right)=0 & \text { in } S:=\mathbb{R} \times \mathbb{R}^{+} \\ U=U_{0} & \text { in } \mathbb{R} \times\{0\},\end{cases}
$$

where $U_{0}$ is a given initial function. Setting $u:=U_{x}$ and $u_{0}:=U_{0}^{\prime}$, the problem is formally transformed in the Cauchy problem for a scalar conservation law,

$$
\begin{cases}u_{t}+[H(u)]_{x}=0 & \text { in } S \\ u=u_{0} & \text { in } \mathbb{R} \times\{0\} .\end{cases}
$$

Problem $(C L)$ was considered in [4, 5] in the context of Radon measure-valued entropy solutions. There it was shown that if

$u_{0}$ is a signed Radon measure on $\mathbb{R}$,

and the singular part $u_{0 s}$ is a finite superposition of Dirac masses,

each initial Dirac mass does not increase in time but, since $H$ is bounded, does not disappear instantaneously, i.e. it survives until a positive waiting time (possibly $\infty$, for example if $H$ is constant). The positivity of the waiting time is in contrast with

Date: August 3, 2020.

1991 Mathematics Subject Classification. 35F21, 35L65, 35D40, 35D99.

Key words and phrases. Hamilton-Jacobi equation, first order hyperbolic conservation laws, singular boundary conditions, waiting time. 
the case of nonlinearities $H$ with superlinear growth, where the regularizing effect is instantaneous [16].

Similarly, in [6] we studied problem $(H J)$ in the context of discontinuous viscosity solutions, and showed that if $H$ is bounded and

$$
U_{0} \text { is piecewise continuous in } \mathbb{R} \text {, with a finite number of jump points, }
$$

the size of each jump discontinuity does not increase in time and does not vanish until a positive waiting time (possibly $\infty$ ). We were motivated by a mathematical model for the process of ion etching, which leads to problem $(H J)$ with bounded and non-convex Hamiltonian $H$ which vanishes at infinity (see [13, 18, 19]) - a set of assumptions scarcely considered in the literature.

In the present paper we are primarily interested in properties of the waiting times. For this purpose it is useful to know that the formal relation $u=U_{x}$ can be made rigorous (if so, the corresponding waiting times for the two problems coincide). We shall prove that this is indeed the case, but, as we explain below, the proof is indirect and based on the existence and uniqueness theory for problems $(C L)$ and $(H J)$. As far as we know, even in the case of non-singular solutions a direct proof, merely based on the definitions of entropy and viscosity solutions, is not available in the literature. We refer to [15] for the indirect approach if $U_{0} \in B V(\mathbb{R})$, and to [7] for the direct approach in the stationary case. In the case of convex nonlinearities $H$, stimulating remarks about the correspondence between viscosity solutions of Hamilton-Jacobi equations and Radon measure-valued solutions of scalar conservation laws can be found in the pioneering paper [10].

The proofs of existence of both a measure-valued solution of problem $(C L)$ and a discontinuous viscosity solution of $(H J)$ are constructive. Choosing suitable approximating problems with smooth initial data $u_{0 n}$ and $U_{0 n}$ (with $U_{0 n}^{\prime}=u_{0 n}$ ) and smooth solutions $u_{n}$ and $U_{n}$, the relation $U_{n x}=u_{n}$ is trivial. Letting $n \rightarrow \infty$, the formal relation between constructed solutions $u$ and $U$ can be made rigorous. So what we need is a uniqueness result for both $u$ and $U$.

Let us point out that suitably defined discontinuous viscosity solutions of $(H J)$ are unique [6], but, as observed in [10, measure-valued entropy solutions of $(C L)$ are not. Only recently an additional compatibility condition (see Definition 3.2 below) was identified which guarantees their uniqueness 4 .

This leads to the following type of result (see Theorem 4.1 for the precise statement). Let $H \in W^{1, \infty}(\mathbb{R})$, let $u_{0}$ be a Radon measure in $\mathbb{R}$ which satisfies (1.1), and let $u$ be a suitably defined measure-valued solution of $(C L)$ which satisfies the compatibility condition. Let $U$ be a suitably defined viscosity solution of $(H J)$ with initial data $U_{0}$ satisfying $U_{0}^{\prime}=u_{0}$ in the sense of measures. Then

$$
\begin{gathered}
U(x, t)=-\int_{0}^{t} H\left(u_{r}(x, s)\right) d s+U_{0}(x) \quad \text { a.e. in } \mathbb{R} \text { for all } t \geq 0, \\
U_{x}=u \text { in } \mathcal{D}^{\prime}(S), \quad u_{s}(\cdot, t)=\sum_{j=1}^{p}\left[U\left(x_{j}^{+}, t\right)-U\left(x_{j}^{-}, t\right)\right] \delta_{x_{j}} \text { for all } t \geq 0,
\end{gathered}
$$

where $x_{1}, \ldots, x_{p}$ are the points where the Dirac masses of $u_{0}$ are concentrated, $u_{r}$ is the density of the absolutely continuous part, $u_{a c}$, of the measure $u$, and $u_{s}$ is the singular part of $u$. Observe that $U_{0}^{\prime}$ is a Radon measure without singular continuous 
part:

$$
U_{0}^{\prime}=\sum_{j=1}^{p}\left[U_{0}\left(x_{j}^{+}\right)-U_{0}\left(x_{j}^{-}\right)\right] \delta_{x_{j}}+\left(U_{0}^{\prime}\right)_{a c} .
$$

Having established the relation between solutions of problems $(H J)$ and $(C L)$, the natural tool to prove properties of the (common) waiting times is the construction of comparison functions. While the comparison principle for viscosity sub- and supersolutions of problem $(H J)$ is known, we shall prove it for entropy solutions of $(C L)$ which satisfy the compatibility condition (Theorem 4.2).

So let $u_{0}=U_{0}^{\prime}$, let $U$ be the unique viscosity solution of $(H J)$ and let $u$ be the unique entropy solution of $(C L)$ which satisfies the compatibility condition. We define the waiting times at the point $x_{i}$ where $U_{0}$ has a jump continuity and where $u_{0 s}$ is concentrated:

$$
\left\{\begin{array}{l}
\tau_{i}:=\sup \left\{\tau>0 ; U(\cdot, t) \text { is discontinuous at } x_{i} \text { for } t \in[0, \tau]\right\} \\
t_{i}:=\sup \left\{\tau>0 ;\left(u_{s}(\cdot, t)\right)\left(\left\{x_{i}\right\}\right) \neq 0 \text { for } t \in[0, \tau]\right\}
\end{array} \quad(i=1, \ldots, p) .\right.
$$

Clearly $\tau_{i}=t_{i}$, and since the comparison principles for the two problems are obviously not equivalent, we can take advantage of the possibility that we can choose to construct comparison functions for problem $(C L)$ or problem $(H J)$ in order to find estimates for the waiting times.

To fix the ideas, we discuss here the case of a positive initial jump at a point $x_{i}$ :

$$
J_{0}\left(x_{i}\right):=U_{0}\left(x_{i}^{+}\right)-U_{0}\left(x_{i}^{-}\right)>0 .
$$

If $H(\xi)$ has no limit at $\infty$, the waiting time is always finite (Theorem 4.4):

$$
\limsup _{\xi \rightarrow \infty} H(\xi)>\liminf _{\xi \rightarrow \infty} H(\xi) \Rightarrow 0<\tau_{i} \leq \frac{J_{0}\left(x_{j}\right)}{\limsup _{\xi \rightarrow \infty} H(\xi)-\liminf _{\xi \rightarrow \infty} H(\xi)} .
$$

If instead

$$
H(\xi) \text { has a limit as } \xi \rightarrow \infty,
$$

it can very well happen that $\tau_{i}=\infty$. It is trivial to see that this can always occur if $H(\xi)$ is constant for sufficiently large $\xi$. So the question is whether $\tau_{i}$ is always finite if

$$
H \text { is not constant in }(c, \infty) \text { for all } c \in \mathbb{R} \text {. }
$$

We are not able to give a definite answer and leave the general question as an open problem. However, we conjecture that this is always the case (for a non definitely constant Hamiltonian), since several partial results in Section 4.3 (see Theorems 4.5. 4.7) give a strong indication in this direction.

The paper is organized as follows. In Section 2 we introduce the basic notations, in Section 3 we review some known results, in Section 4 we present the main results, which are proved in the remaining sections.

\section{Notation}

2.1. Radon measures. For every open subset $\Omega \subseteq \mathbb{R}$ we denote by $C_{c}(\Omega)$ the space of continuous real functions with compact support in $\Omega$ and by $\mathcal{M}^{+}(\Omega)$ the cone of the nonnegative Radon measures on $\Omega$. Following [12, Section 1.3] we say 
that $\mu$ is a (signed) Radon measure on $\Omega$, if there exists $\nu \in \mathcal{M}^{+}(\Omega)$ and a locally $\nu$-summable function $f: \Omega \rightarrow \mathbb{R}$ such that

$$
\mu(K)=\int_{K} f d \nu
$$

for all compact sets $K \subset \Omega$. The space of (signed) Radon measures on $\Omega$ is denoted by $\mathcal{M}(\Omega)$. The measure $\mu \in \mathcal{M}(\Omega)$ is finite if its total variation $|\mu|(\Omega)$ is finite.

If $\mu, \nu \in \mathcal{M}(\Omega)$, we say that $\mu \leq \nu$ in $\mathcal{M}(\Omega)$ if $\nu-\mu \in \mathcal{M}^{+}(\Omega)$. We denote by $\langle\cdot, \cdot\rangle_{\Omega}$ the duality map between $\mathcal{M}(\Omega)$ and $C_{c}(\Omega)$. For any open set $\tilde{\Omega} \subset \subset, \mathcal{M}(\tilde{\Omega})$ is a Banach space with norm $\|\mu\|_{\mathcal{M}(\tilde{\Omega})}:=|\mu|(\tilde{\Omega})$. Similar definitions are used for Radon measures on any subset of $Q:=\Omega \times(0, T)$.

Every $\mu \in \mathcal{M}(\Omega)$ has a unique decomposition $\mu=\mu_{a c}+\mu_{s}$, with $\mu_{a c} \in \mathcal{M}(\Omega)$ absolutely continuous and $\mu_{s} \in \mathcal{M}(\Omega)$ singular with respect to the Lebesgue measure. We denote by $\mu_{r} \in L_{\text {loc }}^{1}(\Omega)$ the density of $\mu_{a c}$. Every function $f \in L_{\text {loc }}^{1}(\Omega)$ can be identified to an absolutely continuous Radon measure on $\Omega$; we shall denote this measure by the same symbol $f$ used for the function.

For every open subset $\Omega \subseteq \mathbb{R}$ we denote by $B V(\Omega)$ the Banach space of functions of bounded variation in $\Omega$ :

$$
B V(\Omega):=\left\{z \in L^{1}(\Omega) \mid z^{\prime} \in \mathcal{M}(\Omega),\left\|z^{\prime}\right\|_{\mathcal{M}(\Omega)}<\infty\right\}, \quad\|z\|_{B V(\Omega)}:=\|z\|_{L^{1}(\Omega)}+\left\|z^{\prime}\right\|_{\mathcal{M}(\Omega)},
$$

where $z^{\prime}$ is the first order distributional derivative. The total variation in $\Omega$ of $z$ is $T V(z ; \Omega):=\left\|z^{\prime}\right\|_{\mathcal{M}(\Omega)}$. We say that $z \in B V_{\text {loc }}(\Omega)$ if $z \in B V(\tilde{\Omega})$ for every open subset $\tilde{\Omega} \subset \subset \Omega$. Similar notions hold if $z \in B V(Q)$; in this case we denote by $z_{x}, z_{t}$ the first order distributional derivatives of $z$.

By $C([0, T] ; \mathcal{M}(\Omega))$ we denote the set of strongly continuous mappings from $[0, T]$ into $\mathcal{M}(\Omega)$ - namely, $u \in C([0, T] ; \mathcal{M}(\Omega))$ if for all $t_{0} \in[0, T]$ and for every compact $K \subset \Omega$ there holds $\left\|u(\cdot, t)-u\left(\cdot, t_{0}\right)\right\|_{\mathcal{M}(K)} \rightarrow 0$ as $t \rightarrow t_{0}$.

We denote by $L_{w *}^{\infty}\left(0, T ; \mathcal{M}^{+}(\Omega)\right)$ the set of nonnegative Radon measures $u \epsilon$ $\mathcal{M}^{+}(S)$ such that for a.e. $t \in(0, T)$ there is a measure $u(\cdot, t) \in \mathcal{M}^{+}(\Omega)$ such that

(i) if $\zeta \in C\left([0, T] ; C_{c}(\Omega)\right)$ the map $t \mapsto\langle u(\cdot, t), \zeta(\cdot, t)\rangle_{\Omega}$ belongs to $L^{1}(0, T)$ and

$$
\langle u, \zeta\rangle_{S}=\int_{0}^{T}\langle u(\cdot, t), \zeta(\cdot, t)\rangle_{\Omega} d t
$$

(ii) the map $t \mapsto\|u(\cdot, t)\|_{\mathcal{M}(K)}$ belongs to $L^{\infty}(0, T)$ for every compact $K \in \Omega$.

By the definition of $L_{w *}^{\infty}\left(0, T ; \mathcal{M}^{+}(\Omega)\right)$, for all $\rho \in C_{c}(\Omega)$ the map $t \mapsto\langle u(\cdot, t), \rho\rangle_{\Omega}$ is measurable, thus the map $u:(0, T) \rightarrow \mathcal{M}^{+}(\Omega)$ is weakly* measurable.

If $u \in L_{w *}^{\infty}\left(0, T ; \mathcal{M}^{+}(\Omega)\right)$, then $u_{a c}, u_{s} \in L_{w *}^{\infty}\left(0, T ; \mathcal{M}^{+}(\Omega)\right), u_{r} \in L^{\infty}\left(0, T ; L_{\mathrm{loc}}^{1}(\Omega)\right)$ and, by (2.1) , for all $\zeta \in C\left([0, T] ; C_{c}(\Omega)\right)$

$$
\left\langle u_{a c}, \zeta\right\rangle_{S}=\iint_{S} u_{r} \zeta d x d t, \quad\left\langle u_{s}, \zeta\right\rangle_{S}=\int_{0}^{T}\left\langle u_{s}(\cdot, t), \zeta(\cdot, t)\right\rangle_{\Omega} d t
$$

Denoting by $[u(\cdot, t)]_{a c},[u(\cdot, t)]_{s} \in \mathcal{M}^{+}(\Omega)$ the absolutely continuous and singular parts of the measure $u(\cdot, t) \in \mathcal{M}^{+}(\Omega)$, a routine proof shows that for a.e. $t \in(0, T)$

$$
u_{s}(\cdot, t)=[u(\cdot, t)]_{s}, \quad u_{a c}(\cdot, t)=[u(\cdot, t)]_{a c}, \quad u_{r}(\cdot, t)=[u(\cdot, t)]_{r},
$$

where $[u(\cdot, t)]_{r}$ denotes the density of the measure $[u(\cdot, t)]_{a c}$.

We say that a (signed) Radon measure $u \in \mathcal{M}(S)$ belongs to $L_{w *}^{\infty}(0, T ; \mathcal{M}(\Omega))$ if both its positive and negative parts $u^{+}$and $u^{-}$belong to $L_{w *}^{\infty}\left(0, T ; \mathcal{M}^{+}(\Omega)\right)$. In particular, this implies that the total variation $|u|$ of the measure $u$ belongs to $L_{w *}^{\infty}\left(0, T ; \mathcal{M}^{+}(\Omega)\right)$, and that conditions $(i)$ and $(i i)$ in the definition of $L_{w *}^{\infty}\left(0, T ; \mathcal{M}^{+}(\Omega)\right)$ hold with $u(\cdot, t):=u^{+}(\cdot, t)-u^{-}(\cdot, t)$ for a.e. $t \in(0, T)$. 
Since $u^{+}$and $u^{-}$are mutually singular, it follows that for a.e. $t$ the nonnegative measures $u^{+}(\cdot, t)$ and $u^{-}(\cdot, t)$ are mutually singular, whence

$$
\begin{gathered}
u^{ \pm}(\cdot, t)=[u(\cdot, t)]^{ \pm}, \quad|u(\cdot, t)|=|u|(\cdot, t) \quad \text { for a.e. } t \in(0, T), \\
u_{s}^{ \pm}(\cdot, t)=[u(\cdot, t)]_{s}^{ \pm}, \quad\left|u_{s}\right|(\cdot, t)=\left|[u(\cdot, t)]_{s}\right| \quad \text { for a.e. } t \in(0, T) .
\end{gathered}
$$

2.2. Functions and envelopes. Let $\chi_{E}$ be the characteristic function of $E \subseteq \mathbb{R}$. For every $u \in \mathbb{R}$ we set

$$
[u]_{ \pm}:=\max \{ \pm u, 0\}, \quad \operatorname{sgn}_{ \pm}(u):= \pm \chi_{\mathbb{R}_{ \pm}}(u), \quad \operatorname{sgn}(u):=\operatorname{sgn}_{-}(u)+\operatorname{sgn}_{+}(u) .
$$

Let $\Omega=(a, b)(-\infty<a<b<\infty)$. We say that a function $f: \Omega \rightarrow \mathbb{R}, f \in L^{\infty}(\Omega)$, is piecewise continuous if:

- $\Omega=\bigcup_{j=1}^{p+1} I_{j}(p \in \mathbb{N})$ with $I_{1}:=\left(a, x_{1}\right), I_{j}:=\left(x_{j-1}, x_{j}\right)$ for $j=2, \ldots, p, I_{p+1}:=\left(x_{p}, b\right)$; - $f_{j}:=f\left\llcorner I_{j}\right.$ admits a representative (denoted again $f_{j}$ for simplicity) which belongs to $C\left(\bar{I}_{j}\right)(j=1, \ldots, p+1) ; f_{j}\left(x_{j}\right) \neq f_{j+1}\left(x_{j}\right)(j=1, \ldots, p)$.

If $\Omega$ is unbounded, $f \in L_{\mathrm{loc}}^{\infty}(\bar{\Omega})$ is piecewise continuous in $\Omega$ if it is piecewise continuous in every bounded interval $\left(a_{0}, b_{0}\right) \subset \Omega$.

Let $Q \subseteq \mathbb{R}^{2}$ be open, $g: Q \mapsto \mathbb{R}$ be a measurable function, $\left(x_{0}, t_{0}\right) \in \bar{Q}$. We set

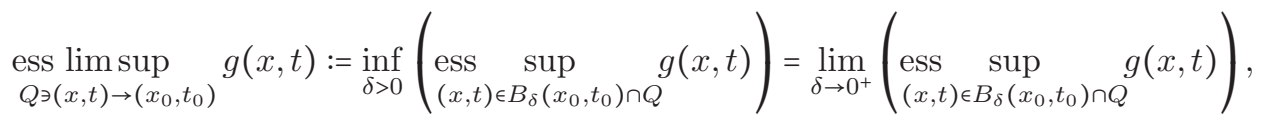

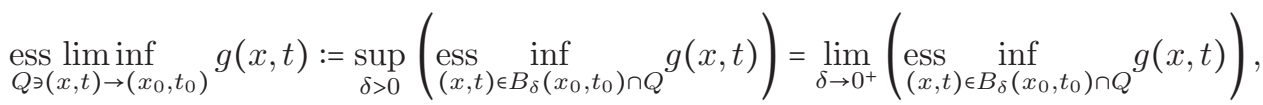

where

$$
B_{r}\left(x_{0}, t_{0}\right):=\left\{(x, t) \in \mathbb{R}^{2} \mid\left(x-x_{0}\right)^{2}+\left(t-t_{0}\right)^{2}<r^{2}\right\} \quad(r>0) .
$$

If ess limsup $\sup _{Q \ni(x, t) \rightarrow\left(x_{0}, t_{0}\right)} g(x, t)=\operatorname{ess}_{\liminf } \operatorname{Qi\ni }_{Q(x, t) \rightarrow\left(x_{0}, t_{0}\right)} g(x, t)$, the essential limit of $g$ at $\left(x_{0}, t_{0}\right)$ is defined as

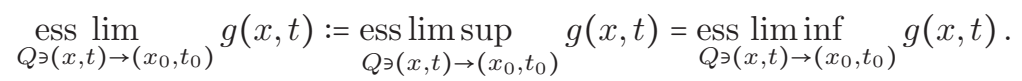

The quantities

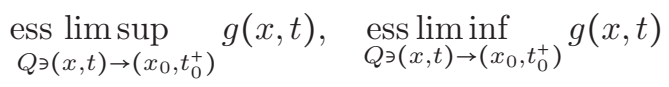

are defined by replacing $B_{r}\left(x_{0}, t_{0}\right)$ by $B_{r}\left(x_{0}, t_{0}\right) \cap\left\{(x, t) \in \mathbb{R}^{2} \mid t>t_{0}\right\}$. Similarly,

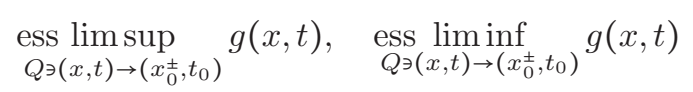

are defined by replacing $B_{r}\left(x_{0}, t_{0}\right)$ by $B_{r}\left(x_{0}, t_{0}\right) \cap\left\{(x, t) \in \mathbb{R}^{2} \mid x>x_{0}\right\}$, respectively by $B_{r}\left(x_{0}, t_{0}\right) \cap\left\{(x, t) \in \mathbb{R}^{2} \mid x<x_{0}\right\}$.

Let $g \in L^{\infty}(Q)$. By the essential upper semicontinuous envelope (shortly, upper envelope) of $g$ we mean the function $g^{*}: \bar{Q} \rightarrow \mathbb{R}$,

$$
g^{*}\left(x_{0}, t_{0}\right):=\underset{Q \ni(x, t) \rightarrow\left(x_{0}, t_{0}\right)}{\operatorname{esslimsup}} g(x, t) \quad \text { for any }\left(x_{0}, t_{0}\right) \in \bar{Q} .
$$

Similarly, the essential lower semicontinuous envelope (shortly, lower envelope) of $g$ is the function $g_{*}: \bar{Q} \rightarrow \mathbb{R}$,

$$
g_{*}\left(x_{0}, t_{0}\right):=\underset{Q \ni(x, t) \rightarrow\left(x_{0}, t_{0}\right)}{\operatorname{ess} \liminf _{\text {inf }}} g(x, t) \quad \text { for any }\left(x_{0}, t_{0}\right) \in \bar{Q} .
$$

Similar definitions hold for measurable functions $f: \mathbb{R} \mapsto \mathbb{R}$. 


\section{DEFINITIONS AND PRELIMINARY RESUlts}

\subsection{Conservation law.}

Definition 3.1. Let $-\infty \leq a<b \leq \infty, \Omega=(a, b), u_{0} \in \mathcal{M}(\Omega)$ and $H \in W^{1, \infty}(\mathbb{R})$. A measure $u \in L_{w *}^{\infty}(0, T ; \mathcal{M}(\Omega))$ is called a solution of

$$
u_{t}+[H(u)]_{x}=0 \quad \text { in } Q:=\Omega \times(0, T), \quad u=u_{0} \quad \text { in } \Omega \times\{0\}
$$

in $Q$ if for all $\zeta \in C^{1}\left([0, T] ; C_{c}^{1}(\Omega)\right), \zeta(\cdot, T)=0$ in $\Omega$ there holds

$$
\iint_{Q}\left[u_{r} \zeta_{t}+H\left(u_{r}\right) \zeta_{x}\right] d x d t+\int_{0}^{T}\left\langle u_{s}(\cdot, t), \zeta_{t}(\cdot, t)\right\rangle_{\Omega} d t=-\left\langle u_{0}, \zeta(\cdot, 0)\right\rangle_{\Omega} .
$$

A solution of (3.1) in $Q$ is called an entropy solution if it satisfies the entropy inequality: for all $k \in \mathbb{R}$ and $\zeta \in C^{1}\left([0, T] ; C_{c}^{1}(\Omega)\right), \zeta \geq 0, \zeta(\cdot, T)=0$ in $\Omega$,

$$
\begin{aligned}
& \iint_{Q}\left\{\left|u_{r}-k\right| \zeta_{t}+\operatorname{sgn}\left(u_{r}-k\right)\left[H\left(u_{r}\right)-H(k)\right] \zeta_{x}\right\} d x d t+ \\
+ & \int_{0}^{T}\left\langle\left|u_{s}(\cdot, t)\right|, \zeta_{t}(\cdot, t)\right\rangle_{\Omega} d t \geq-\int_{\Omega}\left|u_{0 r}(x)-k\right| \zeta(x, 0) d x-\left\langle\left|u_{0 s}\right|, \zeta(\cdot, 0)\right\rangle_{\Omega} .
\end{aligned}
$$

Global (entropy) solutions of (3.1) are (entropy) solutions in $\Omega \times(0, T)$ for all $T>0$.

In particular, setting $\Omega=\mathbb{R}$, we have defined a (global) entropy solution of the Cauchy problem $(C L)$. Summing and subtracting (3.2) and (3.3), we find that entropy solutions $u$ in $Q$ of (3.1) satisfy

$$
\begin{aligned}
& \iint_{Q}\left\{\left[u_{r}-k\right]_{ \pm} \zeta_{t}+\operatorname{sgn}_{ \pm}\left(u_{r}-k\right)\left[H\left(u_{r}\right)-H(k)\right] \zeta_{x}\right\} d x d t+ \\
& \quad+\int_{0}^{T}\left\langle u_{s}^{ \pm}(\cdot, t), \zeta_{t}(\cdot, t)\right\rangle_{\Omega} d t \geq-\int_{\Omega}\left[u_{0 r}(x)-k\right]_{ \pm} \zeta(x, 0) d x-\left\langle u_{0 s}^{ \pm}, \zeta(\cdot, 0)\right\rangle_{\Omega}
\end{aligned}
$$

for all $k \in \mathbb{R}$ and $\zeta \in C^{1}\left([0, T] ; C_{c}^{1}(\Omega)\right), \zeta \geq 0, \zeta(\cdot, T)=0$ in $\Omega$.

Entropy solutions satisfy the following monotonicity result (see [5, Theorem 3.3]).

Theorem 3.1. Let $\left(H_{1}\right)$ hold, let $u_{0} \in \mathcal{M}(\Omega)$ and let $u$ be an entropy solution of (3.1) in $Q$. Then for a.e. $0 \leq t_{1} \leq t_{2} \leq T$

$$
\left[u\left(\cdot, t_{2}\right)\right]_{s}^{ \pm} \leq\left[u\left(\cdot, t_{1}\right)\right]_{s}^{ \pm} \leq u_{0 s}^{ \pm} \quad \text { in } \mathcal{M}(\Omega) .
$$

From now on we consider entropy solutions of (3.1) with initial data $u_{0}$ which satisfy

$\left(H_{2}\right) \quad\left\{\begin{array}{l}u_{0} \text { is a Radon measure on } \Omega, \text { finite if } \Omega \text { is bounded; } \\ u_{0 s}=\sum_{j=1}^{p} c_{j} \delta_{x_{j}} \text { with } x_{1}<x_{2}<\cdots<x_{p}, \quad c_{j} \in \mathbb{R} \backslash\{0\} \text { for } 1 \leq j \leq p .\end{array}\right.$

We shall indicate the support of $u_{0 s}$ by $\mathcal{J}:=\left\{x_{1}, x_{2}, \ldots, x_{p}\right\}$.

Let $\left(H_{1}\right)$ and $\left(H_{2}\right)$ be satisfied. If $u$ is an entropy solution of (3.1) in $Q$, it follows from the proof of [3. Proposition 3.20] that $u \in C([0, T] ; \mathcal{M}(\Omega))$. This implies that if $u$ is a global entropy solution of (3.1) in $Q$, then

$$
t_{j}=\sup \left\{t>0 \mid u_{s}(\cdot, t)\left(\left\{x_{j}\right\}\right) \neq 0\right\}>0 \quad \text { for all } x_{j} \in \mathcal{J}=\left\{x_{1}, x_{2}, \ldots, x_{p}\right\} .
$$

More precisely, $t_{j}$ can be estimated from below (see the proof of [5, Corollary 1]):

$$
t_{j} \geq \frac{\left|u_{0 s}\right|\left(\left\{x_{j}\right\}\right)}{2\|H\|_{\infty}} .
$$


In addition it follows from (3.5) that $\operatorname{supp} u_{s} \subseteq \mathcal{J} \times[0, T]$ and, for all $t \in\left(0, t_{j}\right)$,

$$
u_{s}(\cdot, t)\left(\left\{x_{j}\right\}\right) \begin{cases}>0 & \text { if } c_{j}=u_{0 s}\left(\left\{x_{j}\right\}\right)>0 \\ <0 & \text { if } c_{j}=u_{0 s}\left(\left\{x_{j}\right\}\right)<0 .\end{cases}
$$

Definition 3.2. Let $\left(H_{1}\right)-\left(H_{2}\right)$ hold. An entropy solution $u$ of (3.1) in $Q$ is said to satisfy the compatibility condition at $x_{j} \in \mathcal{J}$ if

(3.9a) ess $\lim _{x \rightarrow x_{j}^{+}} \int_{0}^{t_{j}} \operatorname{sgn}_{ \pm}\left(u_{r}(x, t)-k\right)\left[H\left(u_{r}(x, t)\right)-H(k)\right] \beta(t) d t \leq 0 \quad$ if $\pm c_{j}<0$

(3.9b) ess $\lim _{x \rightarrow x_{j}^{-}} \int_{0}^{t_{j}} \operatorname{sgn}_{ \pm}\left(u_{r}(x, t)-k\right)\left[H\left(u_{r}(x, t)\right)-H(k)\right] \beta(t) d t \geq 0 \quad$ if $\pm c_{j}<0$

for all $k \in \mathbb{R}$ and $\beta \in C_{c}^{1}\left(0, t_{j}\right), \beta \geq 0$, where $t_{j} \in(0, T]$ is defined by (3.6).

By [5, Remark 7] the limits in 3.9a - 3.9b) exist and are finite.

Before stating the basic well-posedness result for the Cauchy problem, we introduce the following singular Cauchy-Dirichlet problems, where $m_{1}, m_{2}= \pm \infty$ :

- if $\Omega=(a, b)$ with $-\infty<a<b<\infty$,

$$
\begin{cases}u_{t}+[H(u)]_{x}=0 & \text { in } Q \\ u=m_{1} & \text { in }\{a\} \times(0, T) \\ u=m_{2} & \text { in }\{b\} \times(0, T) \\ u=u_{0} & \text { in } \Omega \times\{0\} ;\end{cases}
$$

- if $\Omega=(-\infty, b)$ with $b<\infty$,

$(D)_{-}$

$$
\begin{cases}u_{t}+[H(u)]_{x}=0 & \text { in } Q \\ u=m_{2} & \text { in }\{b\} \times(0, T) \\ u=u_{0} & \text { in } \Omega \times\{0\} ;\end{cases}
$$

- if $\Omega=(a, \infty)$ with $a>-\infty$,

$(D)_{+}$

$$
\begin{cases}u_{t}+[H(u)]_{x}=0 & \text { in } Q \\ u=m_{1} & \text { in }\{a\} \times(0, T) \\ u=u_{0} & \text { in } \Omega \times\{0\} .\end{cases}
$$

Definition 3.3. Let $\Omega=(a, b)$ with $-\infty<a<b<\infty$. Let $\left(H_{1}\right)$ hold, and let $u_{0} \in \mathcal{M}(\Omega)$. An entropy solution $u$ of $(D)$ in $Q$ with $m_{1}, m_{2}= \pm \infty$ is an entropy solution of (3.1) in $Q$ such that for all $k \in \mathbb{R}$ and $\beta \in C_{c}^{1}(0, T), \beta \geq 0$ there holds

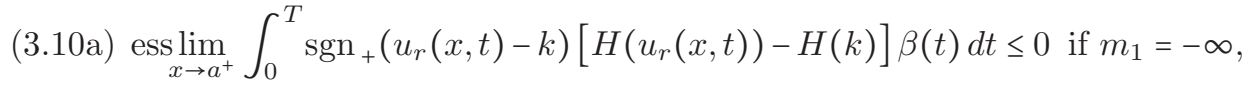

$(3.10 \mathrm{~b}) \underset{x \rightarrow a^{+}}{\operatorname{ess} \lim _{0}} \int_{\operatorname{sgn}}^{T}\left(u_{r}(x, t)-k\right)\left[H\left(u_{r}(x, t)\right)-H(k)\right] \beta(t) d t \leq 0$ if $m_{1}=\infty$,

(3.10c) $\underset{x \rightarrow b^{-}}{\operatorname{ess} \lim _{0}} \int_{0}^{T} \operatorname{sgn}_{+}\left(u_{r}(x, t)-k\right)\left[H\left(u_{r}(x, t)\right)-H(k)\right] \beta(t) d t \geq 0$ if $m_{2}=-\infty$,

(3.10d) $\underset{x \rightarrow b^{-}}{\operatorname{ess} \lim _{0}} \int_{0}^{T} \operatorname{sgn}_{-}\left(u_{r}(x, t)-k\right)\left[H\left(u_{r}(x, t)\right)-H(k)\right] \beta(t) d t \geq 0$ if $m_{2}=\infty$.

Entropy solutions of $(D)_{-}$and $(D)_{+}$are defined by dropping conditions (3.10a)(3.10b) at $x=a(\mathrm{resp}$. (3.10c)-3.10d at $x=b)$. 
Again it follows from [5, Remark 7] that the limits in (3.10) exist and are finite. The proof of the following well-posedness result is basically the same as in the case of problem $(C L)$ (see [5. Theorem 3.5]; for the existence part, see also the proof of Theorem 4.2 below).

Theorem 3.2. Let $\left(H_{1}\right)$ and $\left(H_{2}\right)$ be satisfied. Then the following problems have a unique global entropy solution which satisfies the compatibility condition at all $x_{j} \in \mathcal{J}:$

(i) problem $(D)$, with $m_{1}= \pm \infty, m_{2}= \pm \infty$;

(ii) problem $(D)_{-}$, with $m_{2}= \pm \infty$;

(iii) problem $(D)_{+}$with $m_{1}= \pm \infty$;

(iv) problem $(C L)$.

The following results follow from the proofs of [5, Theorem 3.5 and Proposition 5.8]. The first one states that at the singularities, the one-sided traces of $H(u)=H\left(u_{r}\right)$ at $x_{j} \in \mathcal{J}$ exist in a weak sense:

Proposition 3.3. Let $\left(H_{1}\right)$ and $\left(H_{2}\right)$ be satisfied and let $u$ be the global entropy solution of $(D)$ satisfying the compatibility conditions at all $x_{j} \in \mathcal{J}$. Let $t_{j} \in(0, \infty]$ be defined by (3.6). For all $x_{j}$ there exists $f_{x_{j}^{ \pm}} \in L^{\infty}\left(0, t_{j}\right)$ such that

$$
\text { ess } \lim _{x \rightarrow x_{j}^{ \pm}} \int_{0}^{t_{j}} H(u(x, t)) \beta(t) d t=\int_{0}^{t_{j}} f_{x_{j}^{ \pm}}(t) \beta(t) d t \quad \text { for all } \beta \in C_{c}([0, \infty)) .
$$

Moreover, for a.e. $t \in\left(0, t_{j}\right)$ there holds

$$
\begin{aligned}
& \limsup _{u \rightarrow \infty} H(u) \leq f_{x_{j}^{+}}(t) \leq \sup _{u \in \mathbb{R}} H(u) \quad \text { if } c_{j}>0, \\
& \inf _{u \in \mathbb{R}} H(u) \leq f_{x_{j}^{+}}(t) \leq \liminf _{u \rightarrow-\infty} H(u) \quad \text { if } c_{j}<0, \\
& \inf _{u \in \mathbb{R}} H(u) \leq f_{x_{j}^{-}}(t) \leq \liminf _{u \rightarrow \infty} H(u) \quad \text { if } c_{j}>0, \\
& \limsup _{u \rightarrow-\infty} H(u) \leq f_{x_{j}^{-}}(t) \leq \sup _{u \in \mathbb{R}} H(u) \quad \text { if } c_{j}<0 .
\end{aligned}
$$

The weak traces $f_{x_{j}^{ \pm}}$determine the evolution of the Dirac masses. In fact, since the solution $u$ satisfies the weak formulation (3.2), we have:

Proposition 3.4. Under the assumptions of Proposition 3.3, for all $x_{j} \in \mathcal{J}$,

$$
\begin{gathered}
u_{s}(t)\left\llcorner\left\{x_{j}\right\}=C_{j}(t) \delta_{x_{j}}, \quad C_{j}(t):= \begin{cases}c_{j}-\int_{0}^{t}\left[f_{x_{j}^{+}}(s)-f_{x_{j}^{-}}(s)\right] d s & \text { if } 0 \leq t<t_{j} \\
0 & \text { if } t \geq t_{j},\end{cases} \right. \\
C_{j}(t):=\left\{\begin{array}{ll}
>0 & \text { if } c_{j}>0 \\
<0 & \text { if } c_{j}<0
\end{array} \text { for every } 0 \leq t<t_{j} .\right.
\end{gathered}
$$

Similar results hold for problems $(D)_{-}$and $(D)_{+}$when $\Omega$ is an half-line, and for the Cauchy problem $(C L)$ when $\Omega=\mathbb{R}$. 


\subsection{Hamilton-Jacobi equation.}

Definition 3.4. Let $H \in W^{1, \infty}(\mathbb{R}), E \subseteq \mathbb{R}^{2}$ an open set and $U \in L_{\mathrm{loc}}^{\infty}(\bar{E})$. $U$ is a viscosity solution of the equation $U_{t}+H\left(u_{x}\right)=0$ in $E$, if for all $\varphi \in C^{1}(E)$ :

$\varphi_{t}(x, t)+H\left(\varphi_{x}(x, t)\right) \leq 0$ if $(x, t)$ is a local maximum point of $U^{*}-\varphi$ in $E$;

(3.19) $\varphi_{t}(x, t)+H\left(\varphi_{x}(x, t)\right) \geq 0$ if $(x, t)$ is a local minimum point of $U_{\star}-\varphi$ in $E$.

Definition 3.5. Let $-\infty \leq a<b \leq \infty, \Omega=(a, b), U_{0} \in L_{\mathrm{loc}}^{\infty}(\bar{\Omega})$ and $H \in W^{1, \infty}(\mathbb{R})$. A viscosity solution of

$$
\begin{cases}U_{t}(x, t)+H\left(U_{x}(x, t)\right)=0 & \text { in } Q=\Omega \times(0, T) \\ U(\cdot, 0)=U_{0} & \text { in } \Omega\end{cases}
$$

is a viscosity solution of $U_{t}+H\left(u_{x}\right)=0$ in $Q$ such that

$$
U^{*}(\cdot, 0)=\left(U_{0}\right)^{*}, \quad U_{*}(\cdot, 0)=\left(U_{0}\right)_{*} \text { in } \bar{\Omega} .
$$

Global viscosity solutions of (3.20) are viscosity solutions in $\Omega \times(0, T)$ for all $T>0$.

In particular we have defined a viscosity solution of the Cauchy problem $(H J)$. The singular Dirichlet problems for the conservation law naturally correspond to singular Neumann problems for the Hamilton-Jacobi equation, where $m_{1}, m_{2}= \pm \infty$ :

- if $\Omega=(a, b)$ with $-\infty<a<b<\infty$,

$$
\begin{cases}U_{t}+H\left(U_{x}\right)=0 & \text { in } Q \\ U_{x}=m_{1} & \text { in }\{a\} \times(0, T) \\ U_{x}=m_{2} & \text { in }\{b\} \times(0, T) \\ U=U_{0} & \text { in } \Omega \times\{0\} ;\end{cases}
$$

- if $\Omega=(-\infty, b)$ with $b<\infty$,

$$
\begin{cases}U_{t}+H\left(U_{x}\right)=0 & \text { in } Q \\ U_{x}=m_{2} & \text { in }\{b\} \times(0, T) \\ U=U_{0} & \text { in } \Omega \times\{0\}\end{cases}
$$

- if $\Omega=(a, \infty)$ with $a>-\infty$,

$$
\begin{cases}U_{t}+H\left(U_{x}\right)=0 & \text { in } Q \\ U_{x}=m_{1} & \text { in }\{a\} \times(0, T) \\ U=U_{0} & \text { in } \Omega \times\{0\} .\end{cases}
$$

Definition 3.6. Let $\Omega=(a, b)$ with $-\infty<a<b<\infty$ and $\hat{Q}:=\bar{\Omega} \times(0, T]$. Let $\left(H_{1}\right)$ hold, and let $U_{0} \in L_{\mathrm{loc}}^{\infty}(\bar{\Omega})$. A viscosity solution $U$ of $(N)$ with $m_{1}= \pm \infty, m_{2}= \pm \infty$ is a viscosity solution of (3.20) in $Q$ such that for all $\varphi \in C^{1}(\hat{Q})$ there holds:

(i) if $m_{1}=m_{2}=\infty$ :

(3.22) $\varphi_{t}(a, t)+H\left(\varphi_{x}(a, t)\right) \leq 0$ if $(a, t)$ is a local maximum point of $U^{*}-\varphi$ in $\hat{Q}$,

(3.23) $\varphi_{t}(b, t)+H\left(\varphi_{x}(b, t)\right) \geq 0$ if $(b, t)$ is a local minimum point of $U_{\star}-\varphi$ in $\hat{Q}$;

(ii) if $m_{1}=m_{2}=-\infty$ : 
$\varphi_{t}(a, t)+H\left(\varphi_{x}(a, t)\right) \geq 0$ if $(a, t)$ is a local minimum point of $U_{\star}-\varphi$ in $\hat{Q}$,

$(3.25) \varphi_{t}(b, t)+H\left(\varphi_{x}(b, t)\right) \leq 0$ if $(b, t)$ is a local maximum point of $U^{*}-\varphi$ in $\hat{Q}$;

(iii) if $m_{1}=\infty$ and $m_{2}=-\infty$ and $(a, t)$ and/or $(b, t)$ are local maximum points of $U^{*}-\varphi$ in $\hat{Q}$, then

$$
\left\{\begin{array}{l}
\varphi_{t}(a, t)+H\left(\varphi_{x}(a, t)\right) \leq 0, \\
\varphi_{t}(b, t)+H\left(\varphi_{x}(b, t)\right) \leq 0 ;
\end{array}\right.
$$

(iv) if $m_{1}=-\infty$ and $m_{2}=\infty$ and $(a, t)$ and/or $(b, t)$ are local minimum points of $U_{*}-\varphi$ in $\hat{Q}$, then

$$
\left\{\begin{array}{l}
\varphi_{t}(a, t)+H\left(\varphi_{x}(a, t)\right) \geq 0, \\
\varphi_{t}(b, t)+H\left(\varphi_{x}(b, t)\right) \geq 0 .
\end{array}\right.
$$

Viscosity solutions of $(N)_{-}$and $(N)_{+}$are defined as above, dropping conditions at $x=a$, respectively at $x=b$ in Definition 3.6.

The following well-posedness result holds for $(N)$ ([6, Theorem 3.3 and 3.4]).

Theorem 3.5. Let $\Omega=(a, b)$. Let $\left(H_{1}\right)$ hold, and let $U_{0} \in L_{\mathrm{loc}}^{\infty}(\bar{\Omega})$ be piecewise continuous in $\Omega$ with $\mathcal{J}=\left\{x_{1}, \ldots, x_{p}\right\}$ as the set of jump discontinuities. Then there exists a unique global viscosity solution $U$ of problem $(N)$, with $m_{1}= \pm \infty$, $m_{2}= \pm \infty$. Moreover:

(a) for every $j=1, \ldots, p+1$ the restriction $U\left\llcorner\overline{S_{j}}\right.$ has a continuous representative $\tilde{U}_{j}$ in $\overline{S_{j}}$, with $S_{j}:=I_{j} \times \mathbb{R}^{+}, I_{j}:=\left(x_{j-1}, x_{j}\right), x_{0}:=a, x_{p+1}:=b$;

(b) for every $j=1, \ldots, p$ there exists a unique waiting time $\tau_{j} \in(0, \infty]$ such that

$$
\tilde{U}_{j}\left(x_{j}, t\right) \neq \tilde{U}_{j+1}\left(x_{j}, t\right) \Leftrightarrow t \in\left[0, \tau_{j}\right) .
$$

Similar statements hold for $(N)_{-}$with $m_{2}= \pm \infty$ if $\Omega=(-\infty, b)$ with $b<\infty$, for $(N)_{+}$with $m_{1}= \pm \infty$ if $\Omega=(a, \infty)$ with $a>-\infty$, and for $(H J)$ if $\Omega=\mathbb{R}$.

Remark 3.1. Let $U$ be the global viscosity solution of $(N)$ with initial datum $U_{0}$ as in Theorem 3.5. For all $x_{j} \in \mathcal{J}$ we consider the jumps

$$
J_{0}\left(x_{j}\right):=U_{0}\left(x_{j}^{+}\right)-U_{0}\left(x_{j}^{-}\right), \quad J_{t}\left(x_{j}\right):=U\left(x_{j}^{+}, t\right)-U\left(x_{j}^{-}, t\right) \quad(t>0)
$$

(here $U\left(x_{j}^{+}, t\right)=\tilde{U}_{j+1}\left(x_{j}, t\right)$ and $U\left(x_{j}^{-}, t\right)=\tilde{U}_{j}\left(x_{j}, t\right)$; see Theorem 3.5 $\left.(a)\right)$. By Theorem $3.5(b)$ the jump $J_{t}\left(x_{j}\right)$ persists until the strictly positive waiting time

$$
\tau_{j}=\sup \left\{t \in \mathbb{R}^{+} \mid J_{t}\left(x_{j}\right) \neq 0\right\} \in(0, \infty] .
$$

Moreover, as observed in [6. Remark 3.2], jumps cannot change sign,

$$
J_{t}\left(x_{j}\right)\left\{\begin{array}{ll}
>0 & \text { if } J_{0}\left(x_{j}\right)>0 \\
<0 & \text { if } J_{0}\left(x_{j}\right)<0
\end{array} \quad \text { for all } t \in\left[0, \tau_{j}\right)\right.
$$

and are nonincreasing (in absolute value, [6. Theorem 3.4- $(d)]$ ): for $0 \leq t_{0}<t_{1}<\tau_{j}$ $(3.31)$

$$
\left|J_{t_{1}}\left(x_{j}\right)\right| \leq \begin{cases}\left|J_{t_{0}}\left(x_{j}\right)\right|-\left[\limsup _{\xi \rightarrow \infty} H(\xi)-\liminf _{\xi \rightarrow \infty} H(\xi)\right]\left(t_{1}-t_{0}\right) & \text { if } J_{0}\left(x_{j}\right)>0 \\ \left|J_{t_{0}}\left(x_{j}\right)\right|-\left[\limsup _{\xi \rightarrow-\infty} H(\xi)-\liminf _{\xi \rightarrow-\infty} H(\xi)\right]\left(t_{1}-t_{0}\right) & \text { if } J_{0}\left(x_{j}\right)<0 .\end{cases}
$$




\section{RESUlts}

4.1. Conservation law versus Hamilton-Jacobi equation. The correspondence between the solutions $u$ of $(C L)$ and $U$ of $(H J)$, with $u_{0}=U_{0}^{\prime}$, is a special case $($ set $\Omega=\mathbb{R})$ of the following result. Observe that, in terms of $U_{0}$, hypothesis $\left(H_{2}\right)$ on $u_{0}$ becomes

$\left(H_{3}\right)\left\{\begin{array}{l}U_{0} \in B V_{\mathrm{loc}}(\bar{\Omega}) ; U_{0} \in C(\bar{\Omega}) \text { or } \exists x_{1}<\cdots<x_{p}: U_{0}\left(x_{j}^{+}\right) \neq U_{0}\left(x_{j}^{-}\right) \forall x_{j}, \\ U_{0} \in W_{\mathrm{loc}}^{1,1}\left(\bar{I}_{j}\right), I_{j}=\left(x_{j-1}, x_{j}\right)\left(1 \leq j \leq p+1 ; x_{0}=a, x_{p+1}=b\right) .\end{array}\right.$

Theorem 4.1. Let $\Omega=(a, b)$ with $-\infty<a<b<\infty$, let $\left(H_{1}\right)-\left(H_{3}\right)$ be satisfied and let $\mathcal{J}=\left\{x_{1}, x_{2}, \ldots, x_{p}\right\}$.

(i) Let $u$ be the unique entropy solution of $(D)$ with initial data $u_{0}=U_{0}^{\prime}$ as in (1.5), which satisfies the compatibility condition at all $x_{j} \in \mathcal{J}$. Set

$$
U(\cdot, t):=-\int_{0}^{t} H\left(u_{r}(\cdot, s)\right) d s+U_{0} \quad \text { a.e. in } \Omega \quad(t \in(0, T)) .
$$

Then $U$ is the unique viscosity solution of $(N)$, and $u$ and $U$ satisfy (1.4).

(ii) Let $U$ be the unique viscosity solution of $(N)$. Then the distributional derivative $U_{x}$ belongs to $C([0, T] ; \mathcal{M}(\Omega))$, the measure $u:=U_{x}$ is the unique entropy solution of problem $(D)$ with initial data $u_{0}:=U_{0}^{\prime}$ which satisfies the compatibility condition at all $x_{j} \in \mathcal{J}$, and $u$ and $U$ satisfy (1.3) and (1.4).

Similar statements hold if $\Omega$ is unbounded.

4.2. Comparison. We shall prove the following:

Theorem 4.2. Let $\Omega=(a, b)$ with $-\infty<a<b<\infty$, and let $\left(H_{1}\right)$ hold. Let $u_{0}, v_{0} \in \mathcal{M}(\Omega)$ satisfy

$$
\begin{cases}u_{0 s}=\sum_{j=1}^{p} c_{j} \delta_{x_{j}} & \text { with } x_{1}<x_{2}<\ldots x_{p}, \quad c_{j} \in \mathbb{R} \backslash\{0\} \text { for } 1 \leq j \leq p, \\ v_{0 s}=\sum_{j=1}^{q} d_{j} \delta_{x_{j}^{\prime}} & \text { with } x_{1}^{\prime}<x_{2}^{\prime}<\ldots x_{q}^{\prime},\end{cases}
$$

and let $u_{0} \leq v_{0}$ in $\mathcal{M}(\Omega)$. Let $u, v$ be the entropy solutions of $(D)$ with initial data $u_{0}, v_{0}$ given by Theorem 3.2 (in particular $u$ and $v$ satisfy the compatibility condition). Then $u(\cdot, t) \leq v(\cdot, t)$ in $\mathcal{M}(\Omega)$ for all $t \in[0, T]$.

Similar statements hold if $\Omega$ is unbounded.

The companion result for solutions of $(N)$ is known ([6, Corollary 3.5]):

Theorem 4.3. Let $\Omega=(a, b)$ with $-\infty \leq a<b \leq \infty$, and let $\left(H_{1}\right)$ hold. Let $U_{0}, V_{0} \in$ $L^{\infty}(\Omega), U_{0}$ and $V_{0}$ piecewise continuous in $\Omega$ with a finite number of discontinuities. If $U$ and $V$ are viscosity solutions of problem $(N)$ in $Q$ with initial data $U_{0} \leq V_{0}$ a.e. in $\Omega$, then $U \leq V$ a.e. in $Q$. Similar statements hold if $\Omega$ is unbounded.

Observe that the above assumptions on $U_{0}$ and $V_{0}$ are satisfied if $\left(H_{3}\right)$ holds.

4.3. Waiting time for global solutions of $(H J)$ and $(C L)$. The first result is an upper bound for the waiting times of solutions of problem $(H J)$ if the Hamiltonian $H(\xi)$ does not have a limit as $\xi \rightarrow \pm \infty$.

Theorem 4.4. Let $H \in W^{1, \infty}(\mathbb{R})$ and let $U_{0} \in L_{\mathrm{loc}}^{\infty}(\mathbb{R})$ be piecewise continuous in $\mathbb{R}$ with a finite number of discontinuities: $\mathcal{J}=\left\{x_{1}, \ldots, x_{p}\right\}$. Let

$$
\left(H^{*}\right)_{ \pm}:=\limsup _{\xi \rightarrow \pm \infty} H(\xi), \quad\left(H_{*}\right)_{ \pm}:=\liminf _{\xi \rightarrow \pm \infty} H(\xi)
$$


and let $U$ be the unique global viscosity solution of $(H J)$. Let the initial jump $J_{0}\left(x_{j}\right)$ and the waiting time $\tau_{j} \in(0,+\infty]$ at $x_{j} \in \mathcal{J}$ be defined by (3.28) and (3.29). Then

$$
\tau_{j} \leq \begin{cases}\frac{J_{0}\left(x_{j}\right)}{\left(H^{*}\right)_{+}-\left(H_{*}\right)_{+}} & \text {if } J_{0}\left(x_{j}\right)>0 \text { and }\left(H^{*}\right)_{+}>\left(H_{*}\right)_{+} \\ \frac{\left|J_{0}\left(x_{j}\right)\right|}{\left(H^{*}\right)_{-}-\left(H_{*}\right)_{-}} & \text {if } J_{0}\left(x_{j}\right)<0 \text { and }\left(H^{*}\right)_{-}>\left(H_{*}\right)_{-} .\end{cases}
$$

By assumption $\left(H_{1}\right)$, both $\left(H^{*}\right)_{ \pm}$and $\left(H_{*}\right)_{ \pm}$are finite.

In view of Theorem 4.4 it is natural to seek estimates of $\tau_{j}$ from above assuming

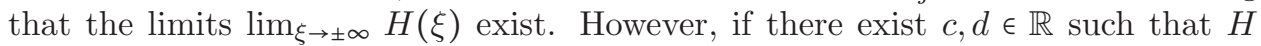
is constant either in $(-\infty, d)$, or in $(c, \infty)$, it is easy to construct examples with $\tau_{j}=\infty$. Hence we make the following assumption:

$$
\left\{\begin{array}{l}
(i) \exists H^{+}:=\lim _{\xi \rightarrow \infty} H(\xi) ; \nexists c>0 \text { such that } H \text { is constant in }(c, \infty) ; \\
\text { (ii) } \exists H^{-}:=\lim _{\xi \rightarrow-\infty} H(\xi) ; \quad \nexists d<0 \text { such that } H \text { is constant in }(-\infty, d) .
\end{array}\right.
$$

Theorem 4.5. Let $\left(H_{1}\right)$ hold. Let $U_{0} \in L_{\text {loc }}^{\infty}(\mathbb{R})$ be piecewise continuous in $\mathbb{R}$, let $\mathcal{J}$ be the finite set of its discontinuities, and let $A, B>0$ be such that

$$
\left|U_{0}(x)\right| \leq A+B|x| \quad \text { for all } x \in \mathbb{R} .
$$

Let $U$ be the unique global viscosity solution of $(H J)$ with initial data $U_{0}$. Then for every $x_{j} \in \mathcal{J}$ the waiting time $\tau_{j}$ is finite if either $J_{0}\left(x_{j}\right)>0$ and $H$ satisfies $\left(H_{4}\right)-(i)$, or $J_{0}\left(x_{j}\right)<0$ and $H$ satisfies $\left(H_{4}\right)-(i i)$.

In view of the correspondence between problems $(H J)$ and $(C L)$ stated in Theorem 4.1, the above results concerning the waiting time have a counterpart for global entropy solutions of $(C L)$. For every $U_{0} \in L_{\text {loc }}^{\infty}(\mathbb{R})$ and $u_{0} \in \mathcal{M}(\mathbb{R})$ as in assumptions $\left(H_{2}\right)-\left(H_{3}\right)$, with $U_{0}^{\prime}=u_{0}$ in $\mathcal{M}(\mathbb{R})$, let $U \in L_{\mathrm{loc}}^{\infty}(\bar{S})$ and $u \in C([0, \infty) ; \mathcal{M}(\mathbb{R}))$ be the global viscosity solution of $(H J)$, respectively the global entropy solution of $(C L)$ satisfying the compatibility condition at every $x_{j} \in \mathcal{J}=\operatorname{supp} u_{0 s}$. Then for every $x_{j} \in \mathcal{J}$

$$
J_{0}\left(x_{j}\right)=u_{0 s}\left(\left\{x_{j}\right\}\right)=c_{j}
$$

and the waiting times for the persistence of jumps in $(H J)$ (see (3.29) ) and of the singular part in $(C L)$ (see (3.6)) coincide, namely

$$
\begin{gathered}
t_{j}=\tau_{j}, \\
u_{s}(\cdot, t)\left(\left\{x_{j}\right\}\right)=J_{t}\left(x_{j}\right) \text { for every } 0 \leq t \leq t_{j}
\end{gathered}
$$

(see (1.4) and (3.28)). Therefore, as a by-product of Theorems 4.1, 4.4 and 4.5 we have the following statements.

Corollary 4.6. Let $\left(H_{1}\right)-\left(H_{2}\right)$ hold. Let $u \in C([0, \infty) ; \mathcal{M}(\mathbb{R}))$ be the unique global entropy solution of $(C L)$ with initial data $u_{0}$, which satisfies the compatibility condition at all $x_{j} \in \mathcal{J}$. Let $t_{j}$ be the waiting time defined by (3.6). Then

$$
t_{j} \leq \begin{cases}\frac{c_{j}}{\left(H^{*}\right)_{+}-\left(H_{*}\right)_{+}} & \text {if } c_{j}>0 \text { and }\left(H^{*}\right)_{+}>\left(H_{*}\right)_{+} \\ \frac{\left|c_{j}\right|}{\left(H^{*}\right)_{-}-\left(H_{*}\right)_{-}} & \text {if } c_{j}<0 \text { and }\left(H^{*}\right)_{-}>\left(H_{*}\right)_{-} .\end{cases}
$$


In addition, if $\bar{A}, \bar{B}>0$ are such that

$$
\left|\int_{0}^{x} u_{0 r}(s) d s\right| \leq \bar{A}+\bar{B}|x| \text { for } x \in \mathbb{R},
$$

then the waiting time $t_{j}$ is finite if either $c_{j}>0$ and $H$ satisfies $\left(H_{4}\right)-(i)$ or $c_{j}<0$ and $\mathrm{H}$ satisfies $\left(\mathrm{H}_{4}\right)-(\mathrm{ii})$.

Remark 4.1. Clearly, assumption $\left(A_{2}\right)$ is satisfied if $u_{0 r} \in L^{1}(\mathbb{R})$ or $u_{0 r} \in L^{\infty}(\mathbb{R})$.

By strengthening the assumptions on $H$, the conclusions in the second part of Corollary 4.6 still hold under very weak assumptions on the initial data. Set

$$
M_{k}^{+}:=\left\|H^{\prime}\right\|_{L^{\infty}(k, \infty)}, \quad M_{k}^{-}:=\left\|H^{\prime}\right\|_{L^{\infty}(-\infty, k)}
$$

(observe that $M_{k}^{ \pm}>0$ by $\left(H_{4}\right)$ ). We introduce the following assumptions:

$$
\left\{\begin{array}{l}
(i) H \text { satisfies }\left(H_{4}\right)-(i), \quad \lim _{k \rightarrow \infty} M_{k}^{+}=0, \quad \limsup _{k \rightarrow \infty} \frac{\left|H(k)-H^{+}\right|}{M_{k}^{+}} \geq C_{0}^{+}>0 ; \\
\text { (ii) } H \text { satisfies }\left(H_{4}\right)-(i i), \quad \lim _{k \rightarrow-\infty} M_{k}^{-}=0, \quad \limsup _{k \rightarrow-\infty} \frac{\left|H(k)-H^{-}\right|}{M_{k}^{-}} \geq C_{0}^{-}>0
\end{array}\right.
$$

(an example of function $H$ satisfying $\left(H_{5}\right)-(i)$ is $H(s)=e^{-s} \sin s$ ), and

$\left(H_{6}\right) \quad \begin{cases}(i) \quad \exists \bar{k}>0 \text { such that either } H(\xi)>H^{+} \text {, or } H(\xi)<H^{+} \text {for any } \xi \geq \bar{k} \\ (\text { ii }) \quad \exists \underline{k}<0 \text { such that either } H(\xi)>H^{-} \text {, or } H(\xi)<H^{-} \text {for any } \xi \leq \underline{k} .\end{cases}$

Theorem 4.7. Let $\left(H_{1}\right)-\left(H_{2}\right)$ hold, and let $u \in C([0, \infty) ; \mathcal{M}(\mathbb{R}))$ be the unique global entropy solution of $(C L)$ with initial data $u_{0}$, which satisfies the compatibility condition at all $x_{j} \in \mathcal{J}$. Then the waiting time $t_{j}$ is finite if either $c_{j}>0$ and $H$ satisfies $\left(H_{5}\right)-(i)$ or $\left(H_{6}\right)-(i)$, or $c_{j}<0$ and $H$ satisfies $\left(H_{5}\right)-(i i)$ or $\left(H_{6}\right)-(i i)$.

Again, by Theorem 4.1 these results for $(C L)$ can be translated to problem $(H J)$.

Corollary 4.8. Let $\left(H_{1}\right)-\left(H_{3}\right)$ hold, and let $U$ be the unique global viscosity solution of $(H J)$ with initial data $U_{0}$. Then for every $x_{j} \in \mathcal{J}$ the waiting time $\tau_{j}$ is finite if either $J_{0}\left(x_{j}\right)>0$ and $H$ satisfies $\left(H_{5}\right)-(i)$ or $\left(H_{6}\right)-(i)$, or $J_{0}\left(x_{j}\right)<0$ and $H$ satisfies $\left(H_{5}\right)-(i i)$ or $\left(H_{6}\right)-(i i)$.

\section{5. ( $D)$ Versus $(N)$ : Proof of Theorem 4.1}

5.1. Preliminary definitions and notations. Let $\Omega=(a, b),-\infty \leq a<b \leq \infty$. Below we generalize problem $(N)$ to the case that $m_{1}, m_{2} \in \overline{\mathbb{R}}:=[-\infty, \infty]$ :

$$
\begin{cases}U_{t}+H\left(U_{x}\right)=0 & \text { in } Q:=\Omega \times(0, T) \\ U_{x}=m_{1} & \text { in }\{a\} \times(0, T) \\ U_{x}=m_{2} & \text { in }\{b\} \times(0, T)\end{cases}
$$

with initial condition

$$
U=U_{0} \quad \text { in } \Omega \times\{0\} .
$$

Definition 5.1. Let $\hat{Q}:=\bar{\Omega} \times(0, T]$ and $m_{1}, m_{2} \in \overline{\mathbb{R}}$.

(i) By a viscosity subsolution of (5.1) in $Q$ we mean any viscosity subsolution $U$ of $U_{t}+H\left(U_{x}\right)=0$ in $Q$ such that if $(a, t)$ and/or $(b, t)$ are local maximum points of $U^{*}-\varphi$ in $\hat{Q}$ for some $\varphi \in C^{1}(\hat{Q})$, then

$$
\begin{cases}\varphi_{t}(a, t)+H\left(\varphi_{x}(a, t)\right) \leq 0 & \text { if } \varphi_{x}(a, t) \leq m_{1} \\ \varphi_{t}(b, t)+H\left(\varphi_{x}(b, t)\right) \leq 0 & \text { if } \varphi_{x}(b, t) \geq m_{2}\end{cases}
$$


(ii) By a viscosity supersolution of (5.1) in $Q$ we mean any viscosity supersolution $U$ of $U_{t}+H\left(U_{x}\right)=0$ in $Q$ such that if $(a, t)$ and/or $(b, t)$ are local minimum points of $U_{*}-\varphi$ in $\hat{Q}$ for some $\varphi \in C^{1}(\hat{Q})$, then

$$
\begin{cases}\varphi_{t}(a, t)+H\left(\varphi_{x}(a, t)\right) \geq 0 & \text { if } \varphi_{x}(a, t) \geq m_{1}, \\ \varphi_{t}(b, t)+H\left(\varphi_{x}(b, t)\right) \geq 0 & \text { if } \varphi_{x}(b, t) \leq m_{2} .\end{cases}
$$

(iii) A function $U$ is called a viscosity solution of (5.1) in $Q$, if it is both a viscosity subsolution and a viscosity supersolution.

(iv) Let $U_{0} \in L_{\mathrm{loc}}^{\infty}(\bar{\Omega})$. A viscosity solution of (5.1) in $Q$ with initial condition (5.2) is a viscosity solution of (5.1) satisfying (3.21).

Remark 5.1. Formally, conditions (5.3) for viscosity subsolutions of (5.1) are void when $m_{1}=-\infty, m_{2}=\infty$; conditions (5.4) for viscosity supersolutions of (5.1) are void when $m_{1}=\infty, m_{2}=-\infty$. Analogously, the boundary conditions at $x=a$ and $x=b$ are dropped if $a=-\infty$ and $b=\infty$, respectively.

5.2. Parabolic approximation. Let $\Omega=(a, b)$ with $-\infty<a<b<\infty$. Let $f_{1, \epsilon}, f_{2, \epsilon}, f_{3, \epsilon} \in C^{\infty}(\mathbb{R})(\epsilon \in(0,1))$ be a partition of unity:

$$
\begin{cases}0 \leq f_{i, \epsilon} \leq 1, \sum_{i=1}^{3} f_{i, \epsilon}=1 \text { in } \mathbb{R}, & \\ f_{1, \epsilon}=1 \text { in }(-\infty, a+2 \sqrt{\epsilon}], & \operatorname{supp} f_{1, \epsilon} \subseteq(-\infty, a+3 \sqrt{\epsilon}], \\ f_{2, \epsilon}=1 \text { in }[a+3 \sqrt{\epsilon}, b-3 \sqrt{\epsilon}], & \operatorname{supp} f_{2, \epsilon} \subseteq[a+2 \sqrt{\epsilon}, b-2 \sqrt{\epsilon}], \\ f_{3, \epsilon}=1 \text { in }[b-2 \sqrt{\epsilon}, \infty), & \operatorname{supp} f_{3, \epsilon} \subseteq[b-3 \sqrt{\epsilon}, \infty),\end{cases}
$$

such that for $i=1,2,3$

$$
\sup _{\epsilon \in(0,1)}\left\|f_{i, \epsilon}^{\prime}\right\|_{L^{1}(\mathbb{R})}<\infty, \quad \sup _{\epsilon \in(0,1)} \sqrt{\epsilon}\left\|f_{i, \epsilon}^{\prime \prime}\right\|_{L^{1}(\mathbb{R})}<\infty .
$$

Let $U_{0} \in C^{\infty}(\bar{\Omega})$ and $m_{1}, m_{2} \in \mathbb{R}$. For every $x \in \bar{\Omega}$, we set

$$
u_{0, \epsilon}:=m_{1} f_{1, \epsilon}+f_{2, \epsilon} U_{0}^{\prime}+m_{2} f_{3, \epsilon}, \quad U_{0, \epsilon}(x):=U_{0}(a)+\int_{a}^{x} u_{0, \epsilon}(s) d s
$$

(to keep notation as simple as possible we suppress the dependence of $u_{0, \epsilon}$ on $m_{1}$, $\left.m_{2}\right)$. Then $U_{0, \epsilon} \in C^{\infty}(\bar{\Omega}), u_{0, \epsilon}=m_{1}$ in $[a, a+\sqrt{\epsilon}], u_{0, \epsilon}=m_{2}$ in $[b-\sqrt{\epsilon}, b]$,

$U_{0, \epsilon}^{\prime}=u_{0, \epsilon}$ in $\bar{\Omega}, \quad\left\|u_{0, \epsilon}\right\|_{L^{\infty}(\Omega)} \leq \max \left\{\left|m_{1}\right|,\left|m_{2}\right|,\left\|U_{0}^{\prime}\right\|_{L^{\infty}(\Omega)}\right\} \quad$ for $\epsilon \in(0,1)$,

$$
\begin{gathered}
\sup _{\epsilon \in(0,1)}\left\|u_{0, \epsilon}^{\prime}\right\|_{L^{1}(\Omega)}<\infty, \quad \sup _{\epsilon \in(0,1)} \sqrt{\epsilon}\left\|u_{0, \epsilon}^{\prime \prime}\right\|_{L^{1}(\Omega)}<\infty, \\
u_{0, \epsilon}(x) \rightarrow U_{0}^{\prime}(x) \quad \text { for all } x \in \Omega, \quad U_{0, \epsilon} \rightarrow U_{0} \quad \text { in } C(\bar{\Omega}), \\
u_{0, \epsilon} \stackrel{*}{\rightarrow} U_{0}^{\prime} \text { in } L^{\infty}(\Omega) \text { and } u_{0, \epsilon} \rightarrow U_{0}^{\prime} \text { in } L^{p}(\Omega) \text { for all } 1 \leq p<\infty .
\end{gathered}
$$

Let $H$ satisfy $\left(H_{1}\right)$. We set

$$
H_{\epsilon}(u):=g_{\epsilon}(u)\left(\left[\eta_{\epsilon} * H\right](u)-\left[\eta_{\epsilon} * H\right](0)\right) \quad(u \in \mathbb{R}),
$$

where $\left\{\eta_{\epsilon}\right\} \subseteq C_{c}^{\infty}(\mathbb{R})$ is a sequence of standard mollifiers and the family $\left\{g_{\epsilon}\right\} \subset$ $C_{c}^{\infty}(\mathbb{R})$ satisfies $g_{\epsilon}=1$ in $(-1 / \epsilon, 1 / \epsilon)$, supp $g_{\epsilon} \subseteq(-2 / \epsilon, 2 / \epsilon)$, and $0 \leq g_{\epsilon} \leq 1,\left|g_{\epsilon}^{\prime}\right| \leq 1$ in $\mathbb{R}$. It is easily seen that

$$
\sup _{\epsilon \in(0,1)}\left\|H_{\epsilon}\right\|_{W^{1, \infty}(\mathbb{R})}<\infty, \quad H_{\epsilon} \rightarrow H \quad \text { uniformly on compact subsets of } \mathbb{R} .
$$


Let $m_{1}, m_{2} \in \mathbb{R}$ and let $u_{\epsilon} \in C^{2,1}(\bar{Q})$ be the unique classical solution (e.g., see 17] of the parabolic problem

$$
\begin{cases}u_{\epsilon t}+\left[H_{\epsilon}\left(u_{\epsilon}\right)\right]_{x}=\epsilon u_{\epsilon x x} & \text { in } Q \\ u_{\epsilon}=m_{1} & \text { in }\{a\} \times(0, T) \\ u_{\epsilon}=m_{2} & \text { in }\{b\} \times(0, T) \\ u_{\epsilon}=u_{0, \epsilon} & \text { in } \Omega \times\{0\} .\end{cases}
$$

By the maximum principle and (5.5) we have

$$
\left\|u_{\epsilon}\right\|_{L^{\infty}(Q)} \leq \max \left\{\left|m_{1}\right|,\left|m_{2}\right|,\left\|U_{0}^{\prime}\right\|_{L^{\infty}(\Omega)}\right\} \quad \text { for any } \epsilon \in(0,1) .
$$

Moreover there exists $c>0$ such that for any $\epsilon \in(0,1)$

$$
\left\|u_{\epsilon x}\right\|_{L^{\infty}\left(0, T ; L^{1}(\Omega)\right)} \leq c, \quad\left\|u_{\epsilon t}\right\|_{L^{\infty}\left(0, T ; L^{1}(\Omega)\right)} \leq c, \quad \epsilon\left\|u_{\epsilon x}\right\|_{L^{\infty}(Q)} \leq c .
$$

In fact, arguing as in the proof of [20, Proposition 3.1] (see also [1]) and using (5.6) we obtain the first two estimates, and the third one easily follows (see [5. Lemma $6.2]$ for details).

By (5.10) the family $\left\{u_{\epsilon}\right\}$ is bounded in $L^{\infty}(Q)$, and $\sup _{\epsilon \in(0,1)}\left\|u_{\epsilon}\right\|_{W^{1,1}(Q)} \leq M$ for some $M>0$. It follows from embedding theorems and the uniqueness of the entropy solution $u \in L^{\infty}\left(0, T ; L^{1}(\Omega)\right)$ of

$$
\left(D_{R}\right), \quad \begin{cases}u_{t}+[H(u)]_{x}=0 & \text { in } Q \\ u=m_{1} & \text { in }\{a\} \times(0, T) \\ u=m_{2} & \text { in }\{b\} \times(0, T) \\ u=U_{0}^{\prime} & \text { in } \Omega \times\{0\}\end{cases}
$$

that

$$
u_{\epsilon} \rightarrow u \quad \text { in } L^{1}(Q) \quad \text { as } \epsilon \rightarrow 0 .
$$

The following result will be used (see [5, Lemma 5.9]).

Lemma 5.1. Let $u$ be given by (5.11). Then for every $t \in(0, T]$

$$
\|u(\cdot, t)\|_{L^{1}(\Omega)} \leq\left\|U_{0}^{\prime}\right\|_{L^{1}(\Omega)}+2\|H\|_{\infty} t .
$$

It is easily seen that the function

$$
U_{\epsilon}(x, t):=-\int_{0}^{t}\left\{H_{\epsilon}\left(u_{\epsilon}(x, s)\right)-\epsilon u_{\epsilon x}(x, s)\right\} d s+U_{0, \epsilon}(x) \quad((x, t) \in \bar{Q})
$$

satisfies $U_{\epsilon x}=u_{\epsilon}$ in $\bar{Q}$ and is the unique classical solution of

$$
\begin{cases}U_{\epsilon t}+H_{\epsilon}\left(U_{\epsilon x}\right)=\epsilon U_{\epsilon x x} & \text { in } Q \\ U_{\epsilon x}=m_{1} & \text { in }\{a\} \times(0, T) \\ U_{\epsilon x}=m_{2} & \text { in }\{b\} \times(0, T) \\ U_{\epsilon}=U_{0, \epsilon} & \text { in } \Omega \times\{0\} .\end{cases}
$$

Then, by (5.10), for all $\epsilon \in(0,1)$ there holds

$$
\begin{aligned}
& \left\|U_{\epsilon x}\right\|_{L^{\infty}(Q)} \leq \max \left\{\left|m_{1}\right|,\left|m_{2}\right|,\left\|U_{0}^{\prime}\right\|_{L^{\infty}(\Omega)}\right\}, \quad\left\|U_{\epsilon x x}\right\|_{L^{\infty}\left(0, T ; L^{1}(\Omega)\right)} \leq c, \\
& \left\|U_{\epsilon x t}\right\|_{L^{\infty}\left(0, T ; L^{1}(\Omega)\right)} \leq c, \quad \epsilon\left\|U_{\epsilon x x}\right\|_{L^{\infty}(Q)} \leq c, \quad\left\|U_{\epsilon t}\right\|_{L^{\infty}(Q)} \leq c+\|H\|_{\infty}
\end{aligned}
$$

(the latter estimate follows from the previous one and the equality $U_{\epsilon t}=\epsilon U_{\epsilon x x}-$ $\left.H_{\epsilon}\left(U_{\epsilon x}\right)\right)$. 
Proposition 5.2. Let $\Omega=(a, b)$ with $-\infty<a<b<\infty, m_{1}, m_{2} \in \mathbb{R}$, and let $\left(H_{1}\right)$ be satisfied. Then for every $U_{0} \in C^{\infty}(\bar{\Omega})$ there exists a viscosity solution of problem (5.1) with initial condition (5.2). Moreover:

(i) $U \in W^{1, \infty}(Q)$ and

$$
\begin{gathered}
\left\|U_{x}\right\|_{L^{\infty}(Q)} \leq \max \left\{\left|m_{1}\right|,\left|m_{2}\right|,\left\|U_{0}^{\prime}\right\|_{L^{\infty}(\Omega)}\right\}, \\
\left\|U_{t}\right\|_{L^{\infty}(Q)} \leq\|H\|_{\infty} .
\end{gathered}
$$

(ii) $U(x, t)=-\int_{0}^{t} H(u(x, s)) d s+U_{0}(x)$ and $U_{x}(x, t)=u(x, t)$ for a.e. $(x, t) \in Q$, where $u$ is the unique entropy solution of problem $\left(D_{R}\right)$.

Proof. By the estimates for $U_{\epsilon x}$ and $U_{\epsilon t}$ in (5.14), the family $\left\{U_{\epsilon}\right\}$ is bounded in $W^{1, \infty}(Q)$. Hence there exist $\left\{U_{\epsilon_{k}}\right\} \subseteq\left\{U_{\epsilon}\right\}$ and $U \in C(\bar{Q})$, with $U_{t}, U_{x} \in L^{\infty}(Q)$, such that $U_{\epsilon_{k}} \rightarrow U$ in $C(\bar{Q})$ (in particular, $U_{\epsilon_{k}}(0)=U_{0, \epsilon_{k}} \rightarrow U_{0}$ in $C(\bar{\Omega})$; see (5.7)), and (5.15a) follows at once from (5.14). Claim (ii) follows from (5.13), the equality $U_{\epsilon x}=u_{\epsilon}$ in $\bar{Q}$, (5.11) and the uniform convergence of $U_{\epsilon_{k}}$ to $U$ in $\bar{Q}$ (observe that, by (5.11) and the last estimate in (5.10), $\epsilon_{k} u_{\epsilon_{k} x} \stackrel{*}{\rightarrow} 0$ in $\left.L^{\infty}(Q)\right)$.

Finally, (5.15b) will follow from (see [6. Proposition 3.2])

$$
\inf _{s \in \mathbb{R}}[-H(s)] \leq \frac{U\left(x, t_{1}\right)-U\left(x, t_{2}\right)}{t_{1}-t_{2}} \leq \sup _{s \in \mathbb{R}}[-H(s)] \quad\left(0<t_{1}<t_{2}<T\right),
$$

as soon as we prove that $U$ is a (continuous) viscosity solution of the equation $U_{t}+H\left(U_{x}\right)=0$ in $Q$. To this purpose, we shall only check conditions (3.18) and (5.3) (checking (3.19) and (5.4) is similar). We distinguish 3 cases: $(\alpha),(\beta),(\gamma)$.

$(\alpha)$ Let $(x, t) \in \Omega \times(0, T]$ be a point where $U-\varphi$, with $\varphi \in C^{2}(\hat{Q})$, has a local maximum. Without loss of generality we may assume that the maximum is strict. Since $U_{\epsilon_{k}} \rightarrow U$ in $C(\bar{Q})$, there exists a sequence $\left\{\left(x_{k}, t_{k}\right)\right\} \subseteq \Omega \times(0, T]$ such that $\left(x_{k}, t_{k}\right) \rightarrow(x, t)$ as $k \rightarrow \infty$, and the function $U_{\epsilon_{k}}-\varphi$ assumes a local maximum at $\left(x_{k}, t_{k}\right) \in \Omega \times(0, T]$. Combined with the regularity of $U_{\epsilon_{k}}$, this implies that

$$
U_{\epsilon_{k} x}\left(x_{k}, t_{k}\right)=\varphi_{x}\left(x_{k}, t_{k}\right), \quad U_{\epsilon_{k} t}\left(x_{k}, t_{k}\right) \geq \varphi_{t}\left(x_{k}, t_{k}\right), \quad U_{\epsilon_{k} x x}\left(x_{k}, t_{k}\right) \leq \varphi_{x x}\left(x_{k}, t_{k}\right),
$$

whence

$$
\begin{aligned}
\varphi_{t}\left(x_{k}, t_{k}\right)+H_{\epsilon_{k}}\left(\varphi_{x}\left(x_{k}, t_{k}\right)\right) & \leq U_{\epsilon_{k} t}\left(x_{k}, t_{k}\right)+H_{\epsilon_{k}}\left(U_{\epsilon_{k} x}\left(x_{k}, t_{k}\right)\right)= \\
& =\epsilon_{k} U_{\epsilon_{k} x x}\left(x_{k}, t_{k}\right) \leq \epsilon_{k} \varphi_{x x}\left(x_{k}, t_{k}\right) .
\end{aligned}
$$

Letting $k \rightarrow \infty$ and using (5.8), we obtain (3.18).

( $\beta)$ Let $U-\varphi\left(\varphi \in C^{2}(\hat{Q})\right)$ assume a strict local maximum at $(a, t), t \in(0, T]$, and let $\varphi_{x}(a, t) \leq m_{1}$. Suppose first that $\varphi_{x}(a, t)<m_{1}$. Arguing as in $(\alpha)$, there exists a sequence $\left\{\left(x_{k}, t_{k}\right)\right\} \subseteq[a, b) \times(0, T]$ such that $\left(x_{k}, t_{k}\right) \rightarrow(a, t)$ as $k \rightarrow \infty$ and $U_{\epsilon_{k}}-\varphi$ assumes a local maximum at $\left(x_{k}, t_{k}\right)$. Observe that $x_{k}>a$ for all $k$, since otherwise $m_{1}=U_{\epsilon_{k} x}\left(a, t_{k}\right) \leq \varphi_{x}\left(a, t_{k}\right)<m_{1}$. So also in this case (5.17) holds, and letting $k \rightarrow \infty$ we obtain the first inequality in (5.3): $\varphi_{t}(a, t)+H\left(\varphi_{x}(a, t)\right) \leq 0$.

Next, let $\varphi_{x}(a, t)=m_{1}$. Set

$$
\varphi_{\delta}(x, t):=\varphi(x, t)-\delta(x-a) \quad((x, t) \in \hat{Q}, \delta>0) ;
$$

notice that $\varphi_{\delta t}=\varphi_{t}, \varphi_{\delta x}=\varphi_{x}-\delta$, and $\varphi_{\delta} \rightarrow \varphi$ in $C(\bar{Q})$ as $\delta \rightarrow 0^{+}$. Since $U-\varphi$ has a strict maximum at $(a, t)$, there exists $\left\{\left(x_{\delta_{j}}, t_{\delta_{j}}\right)\right\} \subset[a, b) \times(0, T]$ such that

$$
\left(x_{\delta_{j}}, t_{\delta_{j}}\right) \rightarrow(a, t), \quad U-\varphi_{\delta_{j}} \text { has a local maximum at }\left(x_{\delta_{j}}, t_{\delta_{j}}\right) .
$$


If $x_{\delta_{j}} \in(a, b)$, as in $(\alpha)$ we obtain that

$$
\varphi_{t}\left(x_{\delta_{j}}, t_{\delta_{j}}\right)+H\left(\varphi_{x}\left(x_{\delta_{j}}, t_{\delta_{j}}\right)-\delta_{j}\right) \leq 0 .
$$

On the other hand, if $x_{\delta_{j}}=a$, for all sufficiently large $j$ we get $t_{\delta_{j}}=t$ (recall that $U-\varphi$ achieves a strict local maximum at the point $(a, t))$, hence $U-\varphi_{\delta_{j}}$ admits a local maximum at the point $(a, t)$. Since $\varphi_{\delta_{j} x}(a, t)=\varphi_{x}(a, t)-\delta_{j}<m_{1}$, by the first part of case $(\beta)$, we get inequality (5.20) in $(a, t)$, namely

$$
\varphi_{t}(a, t)+H\left(\varphi_{x}(a, t)-\delta_{j}\right) \leq 0 .
$$

Letting $j \rightarrow \infty$ in (5.20)-(5.21), the conclusion follows from the continuity of $H$.

$(\gamma)$ If $U-\varphi$ achieves a local maximum at $(b, t)$, with $t \in(0, T]$ and $\varphi_{x}(b, t) \geq m_{2}$, we argue as in step $(\beta)$ and distinguish the cases $\varphi_{x}(b, t)>m_{2}$ and $\varphi_{x}(b, t)=m_{2}$ (we omit the details).

5.3. Proof of the correspondence between problems $(D)$ and $(N)$. We prove Theorem 4.1 first in the case that $u_{0 s}=0$ and $U_{0} \in W_{\mathrm{loc}}^{1,1}(\bar{\Omega})$.

Proposition 5.3. Let $\left(H_{1}\right)$ hold. Let $\Omega=(a, b),-\infty<a<b<\infty, U_{0} \in W^{1,1}(\Omega)$, $u_{0}=U_{0}^{\prime}, m_{1}= \pm \infty$ and $m_{2}= \pm \infty$. Let $U \in C(\bar{Q})$ be the unique viscosity solution of problem $(N)$ and let $u \in C\left([0, T] ; L^{1}(\Omega)\right)$ be the unique entropy solution of problem $(D)$. Then $U \in W^{1,1}(Q)$ and for a.e. $(x, t) \in Q$

$$
U(x, t)=-\int_{0}^{t} H(u(x, s)) d s+U_{0}(x), \quad U_{x}(x, t)=u(x, t) .
$$

Similar statements hold if $\Omega$ is unbounded and $U_{0} \in W_{\mathrm{loc}}^{1,1}(\bar{\Omega})$, with $U \in W_{\mathrm{loc}}^{1,1}(\bar{Q})$.

Proof of Proposition 5.3. The proof consists of several steps.

$\left(\alpha_{1}\right)$ Let $-\infty<a<b<\infty, U_{0} \in C^{\infty}(\bar{\Omega}), m_{1}=\infty$ and $m_{2}=-\infty$ (if $m_{1}, m_{2}= \pm \infty$ the proof is similar). Let $n, p \in \mathbb{N}$ and let $U_{n, p} \in W^{1, \infty}(Q)$ be the viscosity solution of

$$
\left(N_{n, p}\right) \quad \begin{cases}U_{t}+H\left(U_{x}\right)=0 & \text { in } Q \\ U_{x}(a, t)=n, U_{x}(b, t)=-p & \text { if } t \in(0, T) \\ U=U_{0} & \text { in } \Omega \times\{0\}\end{cases}
$$

constructed in Proposition 5.2. Then,

$$
U_{n, p}(x, t)=-\int_{0}^{t} H\left(u_{n, p}(x, s)\right) d s+U_{0}(x), \quad\left[U_{n, p}\right]_{x}(x, t)=u_{n, p}(x, t)
$$

for a.e. $(x, t) \in Q$, where $u_{n, p}$ is the unique entropy solution of

$$
\left(D_{n, p}\right) \quad \begin{cases}{\left[u_{n, p}\right]_{t}+\left[H\left(u_{n, p}\right)\right]_{x}=0} & \text { in } Q \\ u_{n, p}(a, t)=n, u_{n, p}(b, t)=-p & \text { if } t \in(0, T) \\ u_{n, p}=U_{0}^{\prime} & \text { in } \Omega \times\{0\} .\end{cases}
$$

We first let $n \rightarrow \infty$ in the above problems. Observe that

$$
u_{n, p} \rightarrow u_{p} \text { in } L^{1}(Q) \text { as } n \rightarrow \infty,
$$

where $u_{p} \in C\left([0, T] ; L^{1}(\Omega)\right)$ is an entropy solution ([5, proof of Theorem 6.3]) of

$$
\left(D_{\infty, p}\right) \quad \begin{cases}{\left[u_{p}\right]_{t}+\left[H\left(u_{p}\right)\right]_{x}=0} & \text { in } Q \\ u_{p}(a, t)=\infty, u_{p}(b, t)=-p & \text { if } t \in(0, T) \\ u_{p}=U_{0}^{\prime} & \text { in } \Omega \times\{0\} .\end{cases}
$$


In view of (5.23) 1 and (5.15b), $\left\{U_{n, p}\right\}_{n}$ and $\left\{\left(U_{n, p}\right)_{t}\right\}_{n}$ are bounded in $L^{\infty}(Q)$. It follows from (5.23) 2 and (5.24) that $\left\{\left(U_{n, p}\right)_{x}\right\}_{n}$ is bounded in $L^{1}(Q)$ and uniformly integrable. Hence $\left\{U_{n, p}\right\}_{n}$ is uniformly equicontinuous and, possibly up to a subsequence, there exists $U_{p} \in W^{1,1}(Q)$ with $\left(U_{p}\right)_{t} \in L^{\infty}(Q)$ such that

$$
U_{n, p} \rightarrow U_{p} \quad \text { in } C(\bar{Q}) \quad \text { as } n \rightarrow \infty \text {. }
$$

Moreover, by construction, $U_{p}(\cdot, 0)=U_{0}$ in $\Omega,\left(U_{n, p}\right)_{x}=u_{n, p} \rightarrow u_{p}$ in $L^{1}(Q)$,

$$
U_{p}(x, t)=-\int_{0}^{t} H\left(u_{p}(x, s)\right) d s+U_{0}(x), \quad\left(U_{p}\right)_{x}(x, t)=u_{p}(x, t)
$$

for a.e. $(x, t) \in Q($ see (5.23) $-(5.24))$, and, by (5.15b),

$$
\left\|\left(U_{p}\right)_{t}\right\|_{L^{\infty}(Q)} \leq\|H\|_{\infty} .
$$

We claim that $U_{p}$ is a viscosity solution of problem w.15ith $m_{1}=\infty, m_{2}=-p$, i.e.

$$
\left(N_{\infty, p}\right) \quad \begin{cases}\left(U_{p}\right)_{t}+H\left(\left(U_{p}\right)_{x}\right)=0 & \text { in } Q, \\ \left(U_{p}\right)_{x}(a, t)=\infty,\left(U_{p}\right)_{x}(b, t)=-p & \text { if } t \in(0, T), \\ U_{p}=U_{0} & \text { in } \Omega \times\{0\} .\end{cases}
$$

We only check conditions (3.18) and (5.3) (for (3.19) and (5.4) the proof is similar). If $U_{p}-\varphi$ has a strict local maximum at $(x, t) \in \Omega \times(0, T)$, by (5.25) there exists $\left\{\left(x_{n}, t_{n}\right)\right\} \subseteq \Omega \times(0, T)$ such that $\left(x_{n}, t_{n}\right) \rightarrow(x, t)$ and $U_{n, p}-\varphi$ has a local maximum at $\left(x_{n}, t_{n}\right) \in \Omega \times(0, T)$. Since $U_{n, p}$ is a viscosity solution of problem $\left(N_{n, p}\right)$,

$$
\varphi_{t}\left(x_{n}, t_{n}\right)+H\left(\varphi_{x}\left(x_{n}, t_{n}\right)\right) \leq 0 .
$$

If instead $U_{p}-\varphi$ assume a strict local maximum at $(a, t), t \in(0, T)$, we fix a sufficiently small $\delta>0$. Then there exists $\left\{\left(x_{n}, t_{n}\right)\right\} \subseteq[a, b) \times(0, T)$ such that: (i) $\left(x_{n}, t_{n}\right) \rightarrow(a, t)$ as $n \rightarrow \infty, 0<t-\delta \leq t_{n} \leq t+\delta<T$ for all sufficiently large $n$; (ii) $U_{n, p}-\varphi$ achieves a local maximum at $\left(x_{n}, t_{n}\right) ;($ iii $) \varphi_{x}(x, t)<n$ for all $(x, t) \in \bar{\Omega} \times[t-\delta, t+\delta]$. Since $U_{n, p}$ is a viscosity solution of $\left(N_{n, p}\right)$ and $\varphi_{x}\left(x_{n}, t_{n}\right)<n$, we obtain again (5.28). Letting $n \rightarrow \infty$ in (5.28) we obtain the claim. Finally, if $U_{p}-\varphi$ achieves a local maximum at $(b, t)$, with $t \in(0, T)$, the proof is similar.

To conclude step $\left(\alpha_{1}\right)$, we argue as above and let $p \rightarrow \infty$ in problems $\left(D_{\infty, p}\right)$ and $\left(N_{\infty, p}\right)$. More precisely, it can be easily checked that $u_{p} \rightarrow u$ in $L^{1}(Q)$, where $u \in C\left([0, T] ; L^{1}(\Omega)\right)$ is the unique entropy solution of problem $(D)$ with $m_{1}=\infty$, $m_{2}=-\infty$ and $u_{0}=U_{0}^{\prime}$ (see the proof of [5. Theorem 6.3]), and $U_{p} \rightarrow U$ in $C(\bar{Q})$, where $U_{p}$ is the (unique) viscosity solution of the corresponding (singular) Neumann problem $(N)$ with initial condition $U_{0}$. Clearly, by (5.26) and (5.27), it follows that the limiting functions $u$ and $U$ satisfy both (5.22) and the estimate in (5.15b).

$\left(\alpha_{2}\right)$ Let $\Omega=(a, b)$ with $-\infty<a<b<\infty$ and $U_{0} \in W^{1,1}(\Omega)$. Let $\left\{U_{0, k}\right\} \subseteq C^{\infty}(\bar{\Omega})$, $U_{0, k} \rightarrow U_{0}$ in $C(\bar{\Omega})$ as $k \rightarrow \infty$. Let $U_{k}$ be the viscosity solution of problem $(N)$ with $m_{1}= \pm \infty, m_{2}= \pm \infty$ and initial condition $U_{k}(\cdot, 0)=U_{0, k}$, given in step $\left(\alpha_{1}\right)$. Moreover, let $u_{0, k}:=U_{0, k}^{\prime}$, thus $\left\{u_{0, k}\right\} \subseteq B V(\Omega), u_{0, k} \rightarrow U_{0}^{\prime}$ in $L^{1}(\Omega)$ as $k \rightarrow \infty$. Let $\left\{u_{k}\right\}$ be the sequence of entropy solutions to problem $(D)$ with the same boundary conditions $m_{1}= \pm \infty, m_{2}= \pm \infty$ and initial data $u_{0, k}$ considered in step $\left(\alpha_{1}\right)$.

Arguing as in the proof of [5. Theorem 6.3], it can be seen that $u_{k} \rightarrow u$ in $L^{1}(Q)$ as $k \rightarrow \infty$, where $u$ is the entropy solution of problem $(D)$ with initial data $u_{0}=U_{0}^{\prime}$. On the other hand, by [6, Theorem 3.1] there holds

$$
\max _{\bar{Q}}\left|U_{k}-U_{h}\right| \leq \max _{\bar{\Omega}}\left|U_{0, k}-U_{0, h}\right| \quad \text { for all } k, h \in \mathbb{N} \text {. }
$$


Hence $\left\{U_{k}\right\}$ is a Cauchy sequence in $C(\bar{Q})$ and there exists $U \in C(\bar{Q})$ such that $U_{k} \rightarrow U$ in $C(\bar{Q})$. Arguing as in step $\left(\alpha_{1}\right)$ we conclude that $U$ is a viscosity solution of problem $(N)$ with initial condition $U_{0}$.

Finally we observe that (5.22) and (5.15b) are satisfied by $u_{k}, U_{k}$ and $U_{0, k}$ for all $k \in \mathbb{N}$, and so, letting $k \rightarrow \infty$, also by $u$ and $U$. In particular $U \in W^{1,1}(Q)$. This completes the proof of Proposition 5.3 if $\Omega$ is bounded.

$\left(\alpha_{3}\right)$ If $\Omega$ is unbounded, we only the consider the case $\Omega=(a, \infty), a \in \mathbb{R}$ (the other cases are similar). Let $\Omega_{j}:=\left(a, b_{j}\right), b_{j} \leq b_{j+1}$ for every $j \in \mathbb{N}, b_{j} \rightarrow \infty$ as $j \rightarrow \infty$. Let $U_{0} \in C(\bar{\Omega}), U_{0, j} \in C\left(\bar{\Omega}_{j}\right), \operatorname{supp} U_{0, j}=\Omega_{j}$, and let $U_{0, j} \rightarrow U_{0}$ uniformly on compact subsets of $[a, \infty)$. Let $U_{j}$ be the viscosity solution of $(N)$ in $Q_{j}:=\Omega_{j} \times(0, T)$ with initial condition $U_{j}(\cdot, 0)=U_{0, j}$ in $\Omega_{j}$, with the given boundary condition $m_{1}= \pm \infty$ at $\{a\} \times(0, T)$ and arbitrary boundary condition $m_{2}= \pm \infty$ at $\left\{b_{j}\right\} \times(0, T)$. For every $b>a$ set $K:=[a, b] \times[0, T]$, and let $j_{0} \in \mathbb{N}$ be fixed such that $b_{j}>b+\left\|H^{\prime}\right\|_{\infty} T$ for all $j \geq j_{0}$. Applying [6, inequality (3.10) in Theorem 3.1] we obtain, for every $i, j \geq j_{0}$,

$$
\max _{K}\left|U_{j}-U_{i}\right| \leq \max _{\left[a, b+\left\|H^{\prime}\right\|_{\infty} T\right]}\left|U_{0, j}-U_{0, i}\right| .
$$

By the above inequality $\left\{U_{j}\right\}$ is a Cauchy sequence, thus a converging sequence in $C(K)$. Then from the arbitrariness of $K$, by diagonal and separability arguments, there exists a subsequence of $\left\{U_{j}\right\}$ (not relabelled) and $U \in C(\bar{Q})$ such that $U_{j} \rightarrow U$ uniformly on the compact subsets of $\bar{Q}$. Arguing as in step $\left(\alpha_{1}\right)$ it is shown that $U$ is a viscosity solution of problem $\left(N_{+}\right)$with initial data $U_{0}$.

Similarly, let $u \in C\left([0, T] ; L^{1}(\Omega)\right)$ be the unique entropy solution of problem $(D)_{+}$with the same $m_{1}$ as in $(N)_{+}$and initial data $u_{0}=U_{0}^{\prime} \in L_{\text {loc }}^{1}(\bar{\Omega})$. Let $u_{0, j}=$ $U_{0, j}^{\prime}$, thus $u_{0, j} \rightarrow U_{0}^{\prime}$ in $L_{\text {loc }}^{1}(\bar{\Omega})$ as $j \rightarrow \infty$. Let $u_{j}$ be the entropy solution of

$$
\begin{cases}u_{t}+[H(u)]_{x}=0 & \text { in }\left(a, b_{j}\right) \times(0, T) \\ u(a, t)=m_{1}, u\left(b_{j}, t\right)=m_{2} & \text { if } t \in(0, T) \\ u=u_{0, j} & \text { in }\left(a, b_{j}\right) \times\{0\}\end{cases}
$$

with $m_{1}= \pm \infty$ given and $m_{2}= \pm \infty$ fixed as above. Then (up to subsequences) $u_{j} \rightarrow u$ in $L^{\infty}\left(0, T ; L^{1}(\tilde{\Omega})\right)$ for all open intervals $\tilde{\Omega} \subset \subset \bar{\Omega}$ (see the proof of [5, Theorem 6.3]). Since $\tilde{\Omega}$ is bounded, it follows from step $\left(\alpha_{2}\right)$ that for all $j$ large enough there holds

$$
U_{j}(x, t)=-\int_{0}^{t} H\left(u_{j}(x, s)\right) d s+U_{0, j}(x), \quad\left(U_{j}\right)_{x}(x, t)=u_{j}(x, t)
$$

for a.e. $(x, t) \in \tilde{\Omega} \times(0, T))$, and $\left\|\left(U_{j}\right)_{t}\right\|_{L^{\infty}(Q)} \leq\|H\|_{\infty}$. Then letting $j \rightarrow \infty$, it is easily seen that $U \in W_{\mathrm{loc}}^{1,1}(\bar{Q})$ and equality (5.22) follows.

When $\left(H_{2}\right)-\left(H_{3}\right)$ hold, we set $I_{j}=\left(x_{j-1}, x_{j}\right)$ for $j=2, \ldots, p, I_{1}=\left(a, x_{1}\right), I_{p+1}=$ $\left(x_{p}, b\right), Q_{j}=I_{j} \times(0, T)(j=1, \ldots, p+1)$. We denote by $\left(D_{j}\right)$ problem $(D)$ stated in $Q_{j}$ with initial data $u_{0, j}=u_{0}\left\llcorner I_{j} \in L^{1}\left(\bar{I}_{j}\right)\right.$, and by $\left(N_{j}\right)$ problem $(N)$ stated in $Q_{j}$ with initial data $U_{0, j}=U_{0}\left\llcorner I_{j} \in C\left(\overline{I_{j}}\right)\right.$. The proof of the following result can be found in [5, Proposition 5.8].

Proposition 5.4. Let $\left(H_{1}\right)-\left(H_{3}\right)$ hold.

(i) For every $j=2, \ldots, p+1$, let $u_{j}$ be the entropy solution of $\left(D_{j}\right)$ with $m_{1}= \pm \infty$. Then there exists $f_{x_{j-1}^{+}}^{ \pm} \in L^{\infty}(0, T)$ such that for any $\beta \in C_{c}(0, T)$

$$
\text { ess } \lim _{x \rightarrow x_{j-1}^{+}} \int_{0}^{T} H\left(u_{j}(x, t)\right) \beta(t) d t=\int_{0}^{T} f_{x_{j-1}^{+}}^{ \pm}(t) \beta(t) d t .
$$


(ii) For every $j=1, \ldots, p$ let $u_{j}$ be the entropy solution of $\left(D_{j}\right)$ with $m_{2}= \pm \infty$. Then there exists $f_{x_{j}^{-}}^{ \pm} \in L^{\infty}(0, T)$ such that for any $\beta \in C_{c}(0, T)$

$$
\text { ess } \lim _{x \rightarrow x_{j}^{-}} \int_{0}^{T} H\left(u_{j}(x, t)\right) \beta(t) d t=\int_{0}^{T} f_{x_{j}^{-}}^{ \pm}(t) \beta(t) d t .
$$

Moreover, for a.e. $t \in(0, T)$ there holds

$$
\begin{aligned}
& \limsup _{u \rightarrow \infty} H(u) \leq f_{x_{j-1}^{+}}^{+}(t) \leq \sup _{u \in \mathbb{R}} H(u), \\
& \inf _{u \in \mathbb{R}} H(u) \leq f_{x_{j-1}^{+}}^{-}(t) \leq \liminf _{u \rightarrow-\infty} H(u), \\
& \inf _{u \in \mathbb{R}} H(u) \leq f_{x_{j}^{-}}^{+}(t) \leq \liminf _{u \rightarrow \infty} H(u), \\
& \limsup _{u \rightarrow-\infty} H(u) \leq f_{x_{j}^{-}}^{-}(t) \leq \sup _{u \in \mathbb{R}} H(u) .
\end{aligned}
$$

Remark 5.2. By standard density arguments, from (5.29)-(5.30) we get

$$
\text { ess } \lim _{x \rightarrow x_{j-1}^{+}} \int_{0}^{T} H\left(u_{j}(x, t)\right) \zeta(x, t) d t=\int_{0}^{T} f_{x_{j-1}^{+}}^{ \pm}(t) \zeta\left(x_{j-1}, t\right) d t
$$

for all $\zeta \in C^{1}\left([0, T] ; C_{c}^{1}\left(\left[x_{j-1}, x_{j}\right)\right), \zeta(\cdot, 0)=\zeta(\cdot, T)=0\right.$ in $I_{j}$, and

$$
\text { ess } \lim _{x \rightarrow x_{j}^{+}} \int_{0}^{T} H\left(u_{j}(x, t)\right) \zeta(x, t) d t=\int_{0}^{T} f_{x_{j}^{-}}^{ \pm}(t) \zeta\left(x_{j}, t\right) d t
$$

for all $\zeta \in C^{1}\left([0, T] ; C_{c}^{1}\left(\left(x_{j-1}, x_{j}\right]\right), \zeta(\cdot, 0)=\zeta(\cdot, T)=0\right.$ in $I_{j}$.

The following result is an easy consequence of Propositions 5.35.4.

Lemma 5.5. Let $\left(H_{1}\right)-\left(H_{3}\right)$ hold.

(i) Let $j=2, \ldots, p+1$, let $U_{j}$ be the viscosity solution of $\left(N_{j}\right)$ with $m_{1}= \pm \infty$ (and $m_{2}= \pm \infty$ if $j=2, \ldots, p$ ) and initial condition $U_{j}(\cdot, 0)=U_{0, j}$. Let $u_{j}$ be the entropy solution of problem $\left(D_{j}\right)$ with the same boundary conditions and initial data $u_{0, j}=U_{0, j}^{\prime}$. Let $f_{x_{j-1}^{+}}^{ \pm} \in L^{\infty}(0, T)$ be given by Proposition 5.4. Then

$$
U_{j}\left(x_{j-1}, t\right)=-\int_{0}^{t} f_{x_{j-1}^{+}}^{ \pm}(s) d s+U_{0, j}\left(x_{j-1}\right) \quad \text { for all } t \in(0, T] .
$$

(ii) Let $j=1, \ldots, p$, let $U_{j}$ be the viscosity solution of $\left(N_{j}\right)$ with $m_{2}= \pm \infty$ (and $m_{1}= \pm \infty$ if $\left.j=2, \ldots, p\right)$ and initial condition $U_{j}(\cdot, 0)=U_{0, j}$. Let $u_{j}$ be the entropy solution of problem $\left(D_{j}\right)$ with the same boundary conditions and initial data $u_{0, j}=$ $U_{0, j}^{\prime}$. Let $f_{x_{j}^{-}}^{ \pm} \in L^{\infty}(0, T)$ be given by Proposition 5.4. Then

$$
U_{j}\left(x_{j}, t\right)=-\int_{0}^{t} f_{x_{j}^{-}}^{ \pm}(s) d s+U_{0, j}\left(x_{j}\right) \quad \text { for all } t \in(0, T] .
$$

Proof. We only prove $(i)$ with $m_{1}=\infty$. Since $U_{0, j} \in C\left(\bar{I}_{j}\right)$ and $u_{0, j} \in L^{1}\left(\bar{I}_{j}\right)$, (5.34) follows from Proposition 5.3 (5.29) and the essential limit $x \rightarrow x_{j-1}^{+}$in (see (5.22)

$$
U_{j}(x, t)=-\int_{0}^{t} H\left(u_{j}(x, s)\right) d s+U_{0, j}(x) \text { for a.e. } x \in\left(x_{j-1}, x_{j}\right) .
$$


Proof of Theorem 4.1. We rewrite $\left(H_{2}\right)$ as follows:

$$
u_{0 s}=\sum_{j=1}^{p_{+}} c_{j}^{+} \delta_{x_{j}^{\prime}}-\sum_{j=1}^{p_{-}} c_{j}^{-} \delta_{x_{j}^{\prime \prime}} \quad\left(c_{j}^{ \pm} \equiv\left[c_{j}\right]_{ \pm}>0, p_{+}+p_{-}=p\right) .
$$

Since $u_{0}=U_{0}^{\prime}$, by $\left(H_{3}\right)$ there holds (see (1.50)

$$
c_{j}=\mathcal{J}_{0}\left(x_{j}\right):=U_{0}\left(x_{j}^{+}\right)-U_{0}\left(x_{j}^{-}\right)=U_{0, j+1}\left(x_{j}\right)-U_{0, j}\left(x_{j}\right) \quad(j=1, \ldots, p) .
$$

For every $j=1, \ldots, p$ such that $c_{j}=\mathcal{J}_{0}\left(x_{j}\right)>0$ set

$$
C_{j}^{+}(t):=\left[c_{j}-\int_{0}^{t}\left(f_{x_{j}^{+}}^{+}(s)-f_{x_{j}^{-}}^{+}(s)\right) d s\right]_{+} \quad(t \in[0, T]),
$$

with $f_{x_{j}^{+}}^{+}$satisfying (5.29) and $f_{x_{j}^{-}}^{+}$satisfying (5.30); observe that by (5.31a) and (5.31c)

$$
f_{x_{j}^{+}}^{+}(s)-f_{x_{j}^{-}}^{+}(s) \geq 0 \text { for a.e. } s \in(0, T) .
$$

Similarly, for every $j=1, \ldots, p$ such that $c_{j}=\mathcal{J}_{0}\left(x_{j}\right)<0$ set

$$
C_{j}^{-}(t):=\left[c_{j}-\int_{0}^{t}\left(f_{x_{j}^{+}}^{-}(s)-f_{x_{j}^{-}}^{-}(s)\right) d s\right]_{-} \quad(t \in[0, T]),
$$

with $f_{x_{j}^{+}}^{-}$satisfying (5.29) and $f_{x_{j}^{-}}^{-}$satisfying (5.30); observe that by (5.31b) and (5.31d

$$
f_{x_{j}^{+}}^{-}(s)-f_{x_{j}^{-}}^{-}(s) \leq 0 \text { for a.e. } s \in(0, T) .
$$

Moreover, by Proposition 5.3 and (5.34)-5.35) there holds

$$
C_{j}^{ \pm}(t)=\left[U_{j+1}\left(x_{j}, t\right)-U_{j}\left(x_{j}, t\right)\right]_{ \pm} \quad(t \in[0, T]) .
$$

Let $j=1, \ldots, p$ and set

$$
\tau_{1}:=\min \left\{\bar{t}_{1}, \ldots, \bar{t}_{p}\right\}, \quad \text { where } \bar{t}_{j}:=\sup \left\{t \in[0, T] \mid C_{j}^{ \pm}(t)>0\right\} .
$$

Then $\tau_{1}>0$, since $\bar{t}_{j}>0$ and $C_{j}^{ \pm}(0)=c_{j}^{ \pm}>0$. By (5.37)-(5.39) $C_{j}^{ \pm}$is nonincreasing in $(0, T)$, whence $C_{j}^{ \pm}>0$ in $\left[0, \bar{t}_{j}\right)$ and, if $\bar{t}_{j}<T$, there holds $C_{j}^{ \pm}=0$ in $\left[\bar{t}_{j}, T\right]$.

Set $Q_{\tau_{1}}:=\Omega \times\left(0, \tau_{1}\right), Q_{j, \tau_{1}}:=I_{j} \times\left(0, \tau_{1}\right)$. Arguing as in the proof of Theorem 3.2 (see [5, Theorem 3.5]) shows that the unique entropy solution $u \in C\left(\left[0, \tau_{1}\right] ; \mathcal{M}(\Omega)\right)$ of problem $(D)$ in $Q_{\tau_{1}}$ has the following features:

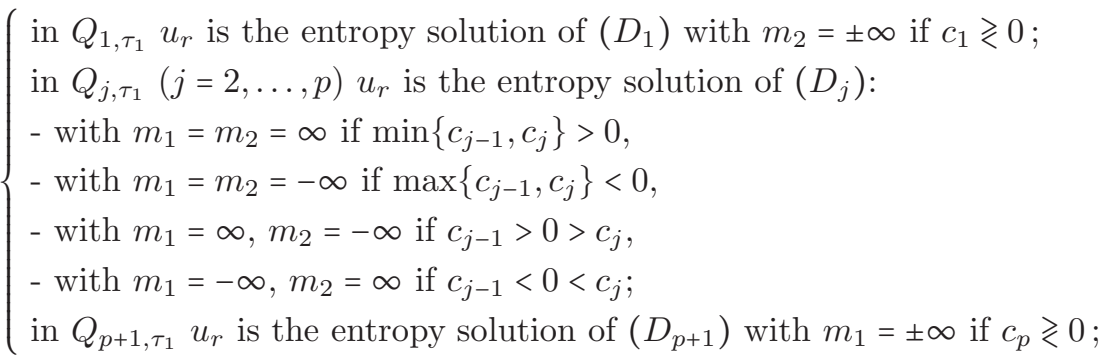

$$
u_{s}(\cdot, t)=\sum_{j=1}^{r} C_{j}^{+}(t) \delta_{x_{j}^{\prime}}-\sum_{j=1}^{s} C_{j}^{-}(t) \delta_{x_{j}^{\prime \prime}}=\sum_{j=1}^{p}\left[U_{j+1}\left(x_{j}, t\right)-U_{j}\left(x_{j}, t\right)\right] \delta_{x_{j}}
$$


(see (5.40) ). Similarly, by the proof of [6. Theorem 3.4] (see also [6, Lemma 5.2]), the unique viscosity solution $U$ of problem $(N)$ in $Q_{\tau_{1}}$ with the same boundary conditions has the following features:

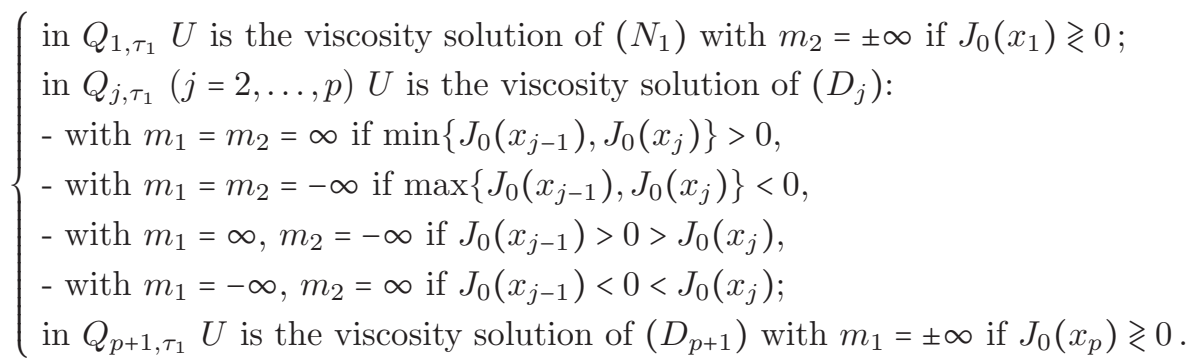

Then, by Proposition 5.3 and (5.42),

- equality (1.3) holds a.e. in $\Omega$ for any $t \in\left[0, \tau_{1}\right]$,

- the second equality in (1.4) holds for any $t \in\left[0, \tau_{1}\right]$.

Let $\rho \in C_{c}^{1}(\Omega)$ and $t \in\left(0, \tau_{1}\right)$. Since

$$
\int_{\Omega} U(x, t) \rho^{\prime}(x) d x=-\int_{0}^{t} \int_{\Omega} H\left(u_{r}(x, s)\right) \rho^{\prime}(x) d x d s-\left\langle u_{0}, \rho\right\rangle_{\Omega}
$$

(see (1.3) ) and

$$
\left\langle u_{0}-u(t), \rho\right\rangle_{\Omega}=-\int_{0}^{t} \int_{\Omega} H\left(u_{r}(x, s)\right) \rho^{\prime}(x) d x d s
$$

(the above equality easily follows by a proper choice of the test function $\zeta$ in the weak formulation (3.2)), we get $\int_{\Omega} U(x, t) \rho^{\prime}(x) d x=-\langle u(t), \rho\rangle_{\Omega}$. Hence

$$
\iint_{Q_{\tau_{1}}} U(x, t) \rho^{\prime}(x) h(t) d x d t=-\int_{0}^{\tau_{1}} h(t)\langle u(t), \rho\rangle_{\Omega} d t=-\langle u, h \rho\rangle_{Q_{\tau_{1}}}
$$

for all $h \in C_{c}^{1}\left(\left(0, \tau_{1}\right)\right)$, which implies that $U_{x}=u$ in $\mathcal{D}^{\prime}\left(Q_{\tau_{1}}\right)$. If $\tau_{1}=T$, the proof is complete. Otherwise, we can repeat the above argument with a lesser number of discontinuities (possibly zero). Hence the conclusion follows.

\section{Comparison: proof of Theorem 4.2}

The proof of Theorem 4.2 relies on some preliminary definitions and results.

6.1. Sub- and supersolutions of $(D)$ with regular initial data. We introduce the notions of sub and supersolutions of problem $(D)$ if $u_{0}$ is a summable function. If $\Omega=(a, b)$ and $-\infty<a<b<\infty$, problem $(D)$ stands for four different initial-boundary value problems, which we denote by $\left(D_{+}^{+}\right),\left(D_{-}^{-}\right),\left(D_{+}^{-}\right)$and $\left(D_{-}^{+}\right)$ according to the four choices $m_{1}=m_{2}=\infty, m_{1}=m_{2}=-\infty, m_{1}=\infty, m_{2}=-\infty$ and $m_{1}=-\infty, m_{2}=\infty$.

Definition 6.1. Let $-\infty<a<b<\infty, \Omega=(a, b)$ and $u_{0} \in L^{1}(\Omega)$, and let $\left(H_{1}\right)$ hold. Let $\underline{u} \in C\left([0, T] ; L^{1}(\Omega)\right)$ satisfy

$$
\lim _{t \rightarrow 0^{+}} \int_{\Omega}\left[\underline{u}(x, t)-u_{0}(x)\right]_{+} d x=0
$$

and, for all $k \in \mathbb{R}$ and $\zeta \in C_{c}^{1}(Q), \zeta \geq 0$ in $Q$,

$$
\iint_{Q}\left\{[\underline{u}-k]_{+} \zeta_{t}+\operatorname{sgn}_{+}(\underline{u}-k)[H(\underline{u})-H(k)] \zeta_{x}\right\} d x d t \geq 0 .
$$

Then $\underline{u}$ is an entropy subsolution of: 
(i) problem $\left(D_{+}^{+}\right)$;

(ii) problem ( $\left.D_{-}^{-}\right)$if for all $k \in \mathbb{R}, \beta \in C_{c}^{1}(0, T), \beta \geq 0$,

$$
\begin{aligned}
& \text { ess } \lim _{\xi \rightarrow a^{+}} \int_{0}^{T} \operatorname{sgn}_{+}(\underline{u}(\xi, t)-k)[H(\underline{u}(\xi, t))-H(k)] \beta(t) d t \leq 0, \\
& \text { ess } \lim _{\eta \rightarrow b^{-}} \int_{0}^{T} \operatorname{sgn}_{+}(\underline{u}(\eta, t)-k)[H(\underline{u}(\eta, t))-H(k)] \beta(t) d t \geq 0 ;
\end{aligned}
$$

(iii) problem $\left(D_{+}^{-}\right)$if $6.1 \mathrm{~b}$ holds for all $k \in \mathbb{R}, \beta \in C_{c}^{1}(0, T), \beta \geq 0$;

(iv) problem ( $\left.D_{-}^{+}\right)$if (6.1a) holds for all $k \in \mathbb{R}, \beta \in C_{c}^{1}(0, T), \beta \geq 0$.

Definition 6.2. Let $-\infty<a<b<\infty, \Omega=(a, b)$ and $u_{0} \in L^{1}(\Omega)$, and let $\left(H_{1}\right)$ hold. Let $\bar{u} \in C\left([0, T] ; L^{1}(\Omega)\right)$ satisfy

$$
\lim _{t \rightarrow 0^{+}} \int_{\Omega}\left[\bar{u}(x, t)-u_{0}(x)\right]_{+} d x=0
$$

and, for all $k \in \mathbb{R}$ and $\zeta \in C_{c}^{1}(Q), \zeta \geq 0$ in $Q$,

$$
\iint_{Q}\left\{[\bar{u}-k]_{-} \zeta_{t}+\operatorname{sgn}_{-}(\bar{u}-k)[H(\bar{u})-H(k)] \zeta_{x}\right\} d x d t \geq 0 .
$$

Then $\bar{u}$ is an entropy supersolution of:

(i) problem $\left(D_{-}^{-}\right)$;

(ii) problem $\left(D_{+}^{+}\right)$if for all $k \in \mathbb{R}$ and $\beta \in C_{c}^{1}(0, T), \beta \geq 0$,

$$
\begin{aligned}
& \text { ess } \lim _{\xi \rightarrow a^{+}} \int_{0}^{T} \operatorname{sgn}(\bar{u}(\xi, t)-k)[H(\bar{u}(\xi, t))-H(k)] \beta(t) d t \leq 0, \\
& \text { ess } \lim _{\eta \rightarrow b^{-}} \int_{0}^{T} \operatorname{sgn} \_(\bar{u}(\eta, t)-k)[H(\bar{u}(\eta, t))-H(k)] \beta(t) d t \geq 0 ;
\end{aligned}
$$

(iii) $\operatorname{problem}\left(D_{+}^{-}\right)$if (6.2a) holds for all $k \in \mathbb{R}, \beta \in C_{c}^{1}(0, T), \beta \geq 0$;

(iv) problem $\left(D_{-}^{+}\right)$if $(6.2 \mathrm{~b})$ holds for all $k \in \mathbb{R}, \beta \in C_{c}^{1}(0, T), \beta \geq 0$.

If $u \in C\left([0, T] ; L^{1}(\Omega)\right)$ is both an entropy subsolution and supersolution of $(D)$, it is an entropy solution in the sense of Definition 3.3. In fact $u$ satisfies the entropy inequalities and it is also a weak solution (see [5, Remark 5]).

Similar definitions hold when $\Omega$ is a half-line and $u_{0} \in L_{\text {loc }}^{1}(\bar{\Omega})$ (see $[5]$ ).

For problem $(D)$ with locally $L^{1}$-initial data the following comparison result holds (see [5, Theorem 5.7]).

Theorem 6.1. Let $\left(H_{1}\right)$ hold and let $u_{0} \in L_{\mathrm{loc}}^{1}(\bar{\Omega})$. Let $\underline{u}, \bar{u} \in C\left([0, T] ; L_{\mathrm{loc}}^{1}(\bar{\Omega})\right)$ be an entropy sub- and supersolution of $(D)$ with the same boundary conditions. Then $\underline{u} \leq \bar{u}$ a.e. in $Q$. In particular, there exists at most one entropy solution of $(D)$.

6.2. Proof of the main result. We prove Theorem 4.2 for problem $(D)$. The proofs for problems $(D)_{ \pm}$and $(C L)$ are similar.

Proposition 6.2. Let $\left(H_{1}\right)$ hold. Let $u_{0}, v_{0} \in \mathcal{M}(\Omega)$ satisfy $\left(H_{2}\right)$, and let $\operatorname{supp} u_{0 s}^{ \pm}=$ $\operatorname{supp} v_{0 s}^{ \pm}$. Let $u, v \in C([0, T] ; \mathcal{M}(\Omega))$ be the entropy solutions of $(D)$ with initial data $u_{0}, v_{0}$ which satisfy the compatibility condition and given by Theorem 3.2. Let $\tau \in(0, T]$ be so small that

$$
\operatorname{supp} u_{s}^{ \pm}(\cdot, t)=\operatorname{supp} v_{s}^{ \pm}(\cdot, t)=\operatorname{supp} u_{0}^{ \pm}=\operatorname{supp} v_{0}^{ \pm} \quad \text { if } 0 \leq t<\tau .
$$

(i) If $u_{0 r} \leq v_{0 r}$ a.e. in $\Omega$, then $u_{r} \leq v_{r}$ a.e. in $Q_{\tau}=\Omega \times(0, \tau)$. 
(ii) Let $f_{x_{j}^{ \pm}}, g_{x_{j}^{ \pm}} \in L^{\infty}(0, \tau)$ be the functions in Proposition 3.3. related to $u$ and $v$, respectively. If $u_{0 r} \leq v_{0 r}$ a.e. in $I_{j}(j=1, \ldots, p+1)$, then

$$
f_{x_{j-1}^{+}} \geq g_{x_{j-1}^{+}} \text {for } j=2, \ldots, p+1, \quad f_{x_{j}^{-}} \leq g_{x_{j}^{-}} \text {for } j=1, \ldots, p \text {, a.e. in }(0, \tau) \text {. }
$$

Proof. (i) By the compatibility conditions (3.9), in each $Q_{j, \tau}:=I_{j} \times(0, \tau)$, with $I_{j}=\left(x_{j-1}, x_{j}\right)\left(j=1, \ldots, p+1 ; x_{0}=a, x_{p+1}=b\right), u_{r, j}:=u_{r}\left\llcorner Q_{j, \tau}\left(\right.\right.$ resp. $v_{r, j}:=$ $v_{r}\left\llcorner Q_{j, \tau}\right)$ is the unique entropy solution of $(D)$ with initial data $u_{0 r, j}:=u_{0 r}\left\llcorner I_{j}\right.$ (resp. $v_{0 r, j}:=v_{0 r}\left\llcorner I_{j}\right.$ ) and $m_{1}= \pm \infty, m_{2}= \pm \infty$ according to the sign of the initial Dirac masses at $x_{j-1}$ and $x_{j}(j=2, \ldots, p)$. Since, by (6.3), $u_{r, j}$ and $v_{r, j}$ satisfy the same boundary conditions and $u_{0 r, j} \leq v_{0 r, j}$ a.e. in $I_{j}$, the conclusion follows from Theorem 6.1.

(ii) First we prove that $f_{x_{j-1}^{+}} \geq g_{x_{j-1}^{+}}$a.e. in $(0, \tau)$. Let $\zeta \in C^{1}\left([0, \tau] ; C_{c}^{1}\left(\left[x_{j-1}, x_{j}\right)\right)\right.$, $\zeta(\cdot, 0)=\zeta(\cdot, \tau)=0$ in $I_{j}$. Arguing as in the proof of [4, Lemma 4.4], we find that

$$
\iint_{Q_{j, \tau}}\left\{\left(u_{r}-k\right) \zeta_{t}+\left[H\left(u_{r}\right)-H(k)\right] \zeta_{x}\right\} d x d t=-\int_{0}^{\tau}\left[f_{x_{j-1}^{+}}(t)-H(k)\right] \zeta\left(x_{j-1}, t\right) d t .
$$

Similarly, if $\zeta \geq 0$ in $Q_{j, \tau}$ it follows from the entropy inequality that

$$
\begin{aligned}
& \iint_{Q_{j, \tau}}\left\{\left|u_{r}-k\right| \zeta_{t}+\operatorname{sgn}\left(u_{r}-k\right)\left[H\left(u_{r}\right)-H(k)\right] \zeta_{x}\right\} d x d t \geq \\
& \quad \geq-\operatorname{ess} \lim _{x \rightarrow x_{j-1}^{+}} \int_{0}^{\tau} \operatorname{sgn}\left(u_{r}(x, t)-k\right)\left[H\left(u_{r}(x, t)\right)-H(k)\right] \zeta(x, t) d t .
\end{aligned}
$$

for all $k \in \mathbb{R}$. Analogous inequalities hold for $v_{r}$.

Since $\operatorname{sgn}(u)=1+2 \operatorname{sgn} n_{-}(u)$ and $\operatorname{sgn}(u)=-1+2 \operatorname{sgn}{ }_{+}(u)$, summing (6.5) and (6.6) it follows from Remark 5.2 that

$$
\begin{aligned}
& \iint_{Q_{j, \tau}}\left\{\left[u_{r}-k\right]_{+} \zeta_{t}+\operatorname{sgn}{ }_{+}\left(u_{r}-k\right)\left[H\left(u_{r}\right)-H(k)\right] \zeta_{x}\right\} d x d t \geq \\
& \geq-\frac{1}{2}\left(\operatorname{ess} \lim _{x \rightarrow x_{j-1}^{+}} \int_{0}^{\tau} \operatorname{sgn}\left(u_{r}(x, t)-k\right)\left[H\left(u_{r}(x, t)\right)-H(k)\right] \zeta(x, t) d t+\right. \\
& \left.\quad+\int_{0}^{\tau}\left[f_{x_{j-1}^{+}}(t)-H(k)\right] \zeta\left(x_{j-1}, t\right) d t\right)= \\
& =-\operatorname{ess} \lim _{x \rightarrow x_{j-1}^{+}} \int_{0}^{\tau} \operatorname{sgn}{ }_{-}\left(u_{r}(x, t)-k\right)\left[H\left(u_{r}(x, t)\right)-H(k)\right] \zeta(x, t) d t- \\
& \quad-\int_{0}^{\tau}\left[f_{x_{j-1}^{+}}(t)-H(k)\right] \zeta\left(x_{j-1}, t\right) d t .
\end{aligned}
$$

Similarly, using again that $\operatorname{sgn}(u)=-1+2 \operatorname{sgn}_{+}(u)$, we obtain

$$
\begin{aligned}
& \iint_{Q_{j, \tau}}\left\{\left[u_{r}-k\right]_{+} \zeta_{t}+\operatorname{sgn}_{+}\left(u_{r}-k\right)\left[H\left(u_{r}\right)-H(k)\right] \zeta_{x}\right\} d x d t \geq \\
& \quad \geq-\operatorname{ess} \lim _{x \rightarrow x_{j-1}^{+}} \int_{0}^{\tau} \operatorname{sgn}_{+}\left(u_{r}(x, t)-k\right)\left[H\left(u_{r}(x, t)\right)-H(k)\right] \zeta(x, t) d t .
\end{aligned}
$$

On the other hand, if we subtract (6.5) from (6.6), we get

$$
\begin{aligned}
& \iint_{Q_{j, \tau}}\left\{\left[u_{r}-k\right]_{-} \zeta_{t}+\operatorname{sgn}_{-}\left(u_{r}-k\right)\left[H\left(u_{r}\right)-H(k)\right] \zeta_{x}\right\} d x d t \geq \\
& \quad \geq- \text { ess } \lim _{x \rightarrow x_{j-1}^{+}} \int_{0}^{\tau} \operatorname{sgn} n_{-}\left(u_{r}(x, t)-k\right)\left[H\left(u_{r}(x, t)\right)-H(k)\right] \zeta(x, t) d t,
\end{aligned}
$$


and

$$
\begin{aligned}
& \iint_{Q_{j, \tau}}\left\{\left[u_{r}-k\right]_{-} \zeta_{t}+\operatorname{sgn} n_{-}\left(u_{r}-k\right)\left[H\left(u_{r}\right)-H(k)\right] \zeta_{x}\right\} d x d t \geq \\
& \geq-\operatorname{ess} \lim _{x \rightarrow x_{j-1}^{+}} \int_{0}^{\tau} \operatorname{sgn}_{+}\left(u_{r}(x, t)-k\right)\left[H\left(u_{r}(x, t)\right)-H(k)\right] \zeta(x, t) d t+ \\
& \quad+\int_{0}^{\tau}\left[f_{x_{j-1}^{+}}(t)-H(k)\right] \zeta\left(x_{j-1}, t\right) d t .
\end{aligned}
$$

Now let $c_{j-1}>0$. From (6.7), (6.9) and the compatibility condition (3.9a) (with $j-1$ instead of $j$ ) we get

$$
\begin{aligned}
& \iint_{Q_{j, \tau}}\left\{\left[u_{r}-k\right]_{+} \zeta_{t}+\operatorname{sgn} n_{+}\left(u_{r}-k\right)\left[H\left(u_{r}\right)-H(k)\right] \zeta_{x}\right\} d x d t \geq \\
& \geq-\int_{0}^{\tau}\left[f_{x_{j-1}^{+}}(t)-H(k)\right] \zeta\left(x_{j-1}, t\right) d t \\
& \iint_{Q_{j, \tau}}\left\{\left[u_{r}-k\right]_{-} \zeta_{t}+\operatorname{sgn}_{-}\left(u_{r}-k\right)\left[H\left(u_{r}\right)-H(k)\right] \zeta_{x}\right\} d x d t \geq 0 .
\end{aligned}
$$

Suppose instead that $c_{j-1}<0$. Then from (6.8), 6.10) and the compatibility condition (3.9a) (with $j-1$ instead of $j$ ) we get

$$
\begin{gathered}
\iint_{Q_{j, \tau}}\left\{\left[u_{r}-k\right]_{+} \zeta_{t}+\operatorname{sgn}_{+}\left(u_{r}-k\right)\left[H\left(u_{r}\right)-H(k)\right] \zeta_{x}\right\} d x d t \geq 0 \\
\iint_{Q_{j, \tau}}\left\{\left[u_{r}-k\right]_{-} \zeta_{t}+\operatorname{sgn} n_{-}\left(u_{r}-k\right)\left[H\left(u_{r}\right)-H(k)\right] \zeta_{x}\right\} d x d t \geq \\
\geq \int_{0}^{\tau}\left[f_{x_{j-1}^{+}}(t)-H(k)\right] \zeta\left(x_{j-1}, t\right) d t
\end{gathered}
$$

Obviously, analogous inequalities hold for $v_{r}$ and $g_{x_{j-1}^{+}}$.

Now we proceed as in the proof of [4, Theorem 3.2] using the Kružkov method of doubling variables. If $c_{j-1}>0$ we use 6.11a and the inequality for $v_{r}=v_{r}(y, s)$ analogous to $6.11 \mathrm{~b}$, namely

$$
\iint_{Q_{j, \tau}}\left\{\left[v_{r}-l\right]_{-} \xi_{s}+\operatorname{sgn}_{-}\left(v_{r}-l\right)\left[H\left(v_{r}\right)-H(l)\right] \xi_{y}\right\} d y d s \geq 0
$$

with $l \in \mathbb{R}$ and $\xi \in C^{1}\left([0, \tau] ; C_{c}^{1}\left(\left[x_{j-1}, x_{j}\right)\right), \xi(\cdot, 0)=\xi(\cdot, \tau)=0\right.$ in $I_{j}, \xi \geq 0$ in $Q_{j, \tau}$. Choose $\psi=\psi(x, t, y, s), \psi \geq 0$ such that $\psi(\cdot, \cdot, y, s), \psi(x, t, \cdot, \cdot) \in C^{1}\left([0, \tau] ; C_{c}^{1}\left(\left[x_{j-1}, x_{j}\right)\right)\right.$, and $\psi(\cdot, 0, \cdot, \cdot)=\psi(\cdot, \tau, \cdot, \cdot)=\psi(\cdot, \cdot, \cdot, 0)=\psi(\cdot, \cdot, \cdot, \tau)=0$ in $I_{j}$. Setting in (6.11a $k=v_{r}(y, s), \zeta=\psi(\cdot, \cdot, y, s)$ we have

$$
\begin{gathered}
\iint_{Q_{j, \tau}}\left\{\operatorname{sgn}_{+}\left(u_{r}(x, t)-v_{r}(y, s)\right)\left[H\left(u_{r}(x, t)\right)-H\left(v_{r}(y, s)\right)\right] \psi_{x}(x, t, y, s)+\right. \\
\left.+\left[u_{r}(x, t)-v_{r}(y, s)\right]_{+} \psi_{t}(x, t, y, s)\right\} d x d t \geq \\
\geq-\int_{0}^{\tau}\left[f_{x_{j-1}^{+}}(t)-H\left(v_{r}(y, s)\right)\right] \psi\left(x_{j-1}, t, y, s\right) d t,
\end{gathered}
$$

whereas from w6.13) with $l=u_{r}(x, t), \xi=\psi(x, t \cdot, \cdot)$, using the identities $[u]_{-}=[-u]_{+}$, $\operatorname{sgn} n_{-}(-u)=-\operatorname{sgn}_{+}(u)$ we get

$$
\begin{gathered}
\iint_{Q_{j, \tau}}\left\{\operatorname{sgn}_{+}\left(u_{r}(x, t)-v_{r}(y, s)\right)\left[H\left(u_{r}(x, t)\right)-H\left(v_{r}(y, s)\right)\right] \psi_{y}(x, t, y, s)+\right. \\
\left.+\left[u_{r}(x, t)-v_{r}(y, s)\right]_{+} \psi_{s}(x, t, y, s)\right\} d y d s \geq 0 .
\end{gathered}
$$


Now choose

$$
\psi(x, t, y, s)=\eta\left(\frac{x+y}{2}, \frac{t+s}{2}\right) \rho_{\epsilon}(x-y) \rho_{\epsilon}(t-s)
$$

where $\eta \in C^{1}\left([0, \tau] ; C_{c}^{1}\left(\left[x_{j-1}, x_{j}\right)\right), \eta \geq 0, \eta(\cdot, 0)=\eta(\cdot, \tau)=0\right.$ in $I_{j}$, and $\rho_{\epsilon}(\epsilon>0)$ is a symmetric mollifier in $\mathbb{R}$. Arguing as in the proof of [4, Theorem 3.2], from the above inequalities we get

$$
\begin{aligned}
& \iint_{Q_{j, \tau}}\left\{\operatorname{sgn}_{+}\left(u_{r}(x, t)-v_{r}(x, t)\right)\left[H\left(u_{r}(x, t)\right)-H\left(v_{r}(x, t)\right)\right] \eta_{x}+\right. \\
& \left.\left.\left[u_{r}(x, t)-v_{r}(x, t)\right]_{+} \eta_{t}\right\} d x d t \geq-\frac{1}{2} \int_{0}^{\tau}\left[f_{x_{j-1}^{+}}(t)-g_{x_{j-1}^{+}}(t)\right)\right] \eta\left(x_{j-1}, t\right) d t .
\end{aligned}
$$

Recalling that if $u_{0 r, j+1} \leq v_{0 r, j+1}$ a.e. in $I_{j}$ then, by part $(i), u_{r, j+1} \leq v_{r, j+1}$ a.e. in $Q_{j, \tau}$, we obtain from (6.14) and the arbitrariness of $\eta$ that $f_{x_{j-1}^{+}} \geq g_{x_{j-1}^{+}}$a.e. in $(0, \tau)$.

If $c_{j-1}<0$ we use (6.12a) and the inequality for $v_{r}=v_{r}(y, s)$ analogous to (6.12b),

$$
\begin{aligned}
& \iint_{Q_{j, \tau}}\left\{\left[v_{r}-l\right]_{-} \xi_{s}+\operatorname{sgn}_{-}\left(v_{r}-l\right)\left[H\left(v_{r}\right)-H(l)\right] \xi_{y}\right\} d y d s \geq \\
& \geq \int_{0}^{\tau}\left[g_{x_{j-1}^{+}}(s)-H(l)\right] \xi\left(x_{j-1}, s\right) d s
\end{aligned}
$$

with $l \in \mathbb{R}$ and $\xi$ as above. Choosing in 6.12a $k=v_{r}(y, s), \zeta=\psi(\cdot, \cdot, y, s)$ with $\psi$ as above gives

$$
\begin{gathered}
\iint_{Q_{j, \tau}}\left\{\operatorname{sgn}_{+}\left(u_{r}(x, t)-v_{r}(y, s)\right)\left[H\left(u_{r}(x, t)\right)-H\left(v_{r}(y, s)\right)\right] \psi_{x}(x, t, y, s)+\right. \\
\left.+\left[u_{r}(x, t)-v_{r}(y, s)\right]_{+} \psi_{t}(x, t, y, s)\right\} d x d t \geq 0 .
\end{gathered}
$$

On the other hand, from (6.15) with $l=u_{r}(x, t), \xi=\psi(x, t \cdot, \cdot)$, using again the identities $[u]_{-}=[-u]_{+}, \operatorname{sgn}_{-}(-u)=-\operatorname{sgn}_{+}(u)$ we get

$$
\begin{gathered}
\iint_{Q_{j, \tau}}\left\{\operatorname{sgn}_{+}\left(u_{r}(x, t)-v_{r}(y, s)\right)\left[H\left(u_{r}(x, t)\right)-H\left(v_{r}(y, s)\right)\right] \psi_{y}(x, t, y, s)+\right. \\
\left.+\left[u_{r}(x, t)-v_{r}(y, s)\right]_{+} \psi_{s}(x, t, y, s)\right\} d y d s \geq \\
\geq \int_{0}^{\tau}\left[g_{x_{j-1}^{+}}(s)-H\left(u_{r}(x, t)\right)\right] \psi\left(x_{j-1}, t, y, s\right) d s .
\end{gathered}
$$

Then arguing as in the proof of (6.14) we get inequality (6.14) for any $\eta$ as above, whence $f_{x_{j-1}^{+}} \geq g_{x_{j-1}^{+}}$a.e. in $(0, \tau)$.

Concerning the inequalities $f_{x_{j}^{-}} \leq g_{x_{j}^{-}}(j=1, \ldots, p)$ a.e. in $(0, \tau)$, the proof relies on the following counterpart of (6.5)-

$$
\begin{gathered}
\iint_{Q_{j, \tau}}\left\{\left(u_{r}-k\right) \zeta_{t}+\left[H\left(u_{r}\right)-H(k)\right] \zeta_{x}\right\} d x d t=\int_{0}^{\tau}\left[f_{x_{j}^{-}}(t)-H(k)\right] \zeta\left(x_{j}, t\right) d t, \\
\iint_{Q_{j, \tau}}\left\{\left|u_{r}-k\right| \zeta_{t}+\operatorname{sgn}\left(u_{r}-k\right)\left[H\left(u_{r}\right)-H(k)\right] \zeta_{x}\right\} d x d t \geq \\
\geq \operatorname{ess} \lim _{x \rightarrow x_{j}^{-}} \int_{0}^{\tau} \operatorname{sgn}\left(u_{r}(x, t)-k\right)\left[H\left(u_{r}(x, t)\right)-H(k)\right] \zeta(x, t) d t
\end{gathered}
$$

where $\zeta \in C^{1}\left([0, \tau] ; C_{c}^{1}\left(\left(x_{j-1}, x_{j}\right]\right), \zeta \geq 0, \zeta(\cdot, 0)=\zeta(\cdot, \tau)=0\right.$ in $I_{j}$, and on the compatibility condition (3.9b). We leave the details to the reader.

Now we can prove Theorem 4.2 .

Proof of Theorem 4.2. Let

$$
\tau=\sup \left\{t \in(0, T) ; \operatorname{supp} u_{s}(t)=\operatorname{supp} u_{0 s}, \operatorname{supp} v_{s}(t)=\operatorname{supp} v_{0 s}\right\} .
$$


Set

$$
\begin{gathered}
\operatorname{supp} u_{0 s} \cup \operatorname{supp} v_{0 s} \equiv\left\{y_{1}, \ldots, y_{r}\right\} \quad \text { with } y_{1}<y_{2}<\ldots<y_{r}, \\
u_{0 s}=\sum_{k=1}^{r} \hat{c}_{k} \delta_{y_{k}}, v_{0 s}=\sum_{k=1}^{r} \hat{d}_{k} \delta_{y_{k}}
\end{gathered}
$$

with $\hat{c}_{k}, \hat{d}_{k} \in \mathbb{R}$, at least one of $\hat{c}_{k}, \hat{d}_{k}$ different from zero, $\hat{c}_{k} \leq \hat{d}_{k}$; observe that

$$
\hat{c}_{k} \hat{d}_{k} \neq 0 \Leftrightarrow y_{k} \in \operatorname{supp} u_{0 s} \cap \operatorname{supp} v_{0 s} \quad(k=1, \ldots, r) .
$$

Also set $I_{k}=\left(y_{k-1}, y_{k}\right)$, with $y_{0}=a, y_{r+1}=b, Q_{k, \tau}=I_{k} \times(0, \tau)$, and $u_{0 r, k}=u_{0 r}\left\llcorner I_{k}\right.$, $v_{0 r, k}=v_{0 r}\left\llcorner I_{k}, u_{r, k}=u_{r}\left\llcorner Q_{k, \tau}, v_{r, k}=v_{r}\left\llcorner Q_{k, \tau}(k=1, \ldots, r+1)\right.\right.\right.$.

By assumption there holds $u_{0 r} \leq v_{0 r}$ a.e. in $I_{k}$ for any $k$. We claim that

$$
u_{r} \leq v_{r} \quad \text { in } Q_{k, \tau} \text { for all } k=1, \ldots, r+1 .
$$

Observe that at each point $y_{k}$ there holds either $\hat{c}_{k} \hat{d}_{k} \leq 0$, or $\hat{c}_{k} \hat{d}_{k}>0$. If $\hat{c}_{k} \hat{d}_{k}=$ $u_{0 s}\left(\left\{y_{k}\right\}\right) v_{0 s}\left(\left\{y_{k}\right\}\right) \leq 0$, by (3.5) there holds $u_{s}(\cdot, t)\left(\left\{y_{k}\right\}\right) \leq 0 \leq v_{s}(\cdot, t)\left(\left\{y_{k}\right\}\right)$ for any $t \in(0, \tau)$, thus in this case

$$
u_{s}(\cdot, t)\left\llcorner\left\{y_{k}\right\} \leq v_{s}(\cdot, t)\left\llcorner\left\{y_{k}\right\} \text { for any } t \in(0, \tau) .\right.\right.
$$

On the other hand, if $\hat{c}_{k} \hat{d}_{k}>0$, there holds either $\hat{c}_{k}>0, \hat{d}_{k}>0$, or $\hat{c}_{k}<0, \hat{d}_{k}<0$. By Proposition 3.4 for any $t \in(0, \tau)$ there holds

$$
u_{s}(\cdot, t)\left\llcorner\left\{y_{k}\right\}=C_{k}(t) \delta_{y_{k}}, \quad v_{s}(\cdot, t)\left\llcorner\left\{y_{k}\right\}=D_{k}(t) \delta_{y_{k}},\right.\right.
$$

where $C_{k}$ are defined by (3.16), and $D_{k}$ are the analogous quantities for $v_{s}$. Assuming $u_{r} \leq v_{r}$ in $Q_{k, \tau}$ and arguing as in the proof of Proposition 6.2 $(i i)$, it is easily seen that inequalities (6.4) hold (with $x_{k}^{+}$instead of $x_{j-1}^{+}$) for any $t \in(0, \tau)$, whence in both cases $\hat{c}_{k}, \hat{d}_{k}>0$ or $\hat{c}_{k}, \hat{d}_{k}<0$ we get

$$
C_{k}(t) \leq D_{k}(t) \text { for all } t \in[0, \tau) .
$$

From (6.18) and (6.19) we obtain (6.17) also in this case. Then by (6.16) and (6.17) there holds $u(\cdot, t) \leq v(\cdot, t)$ in $\mathcal{M}(\Omega)$ for any $t \in[0, \tau]$.

If $\tau=T$ the proof is complete. Otherwise, we can repeat the above arguments in $\Omega \times[\tau, T]$, since we proved that $u(\cdot, \tau) \leq v(\cdot, \tau)$ in $\mathcal{M}(\Omega)$. In a finite time of steps the conclusion follows.

It remains to prove the claim (6.16). We only consider the case that $k=2, \ldots, r$, the proof being simpler for $k=1$ or $r+1$. We distinguish the following cases:

(a) $\hat{c}_{k-1} \hat{d}_{k-1}>0, \hat{c}_{k} \hat{d}_{k}>0$. In this case $u_{r}$ and $v_{r}$ are solutions of the same problem $\left(D_{k}\right) \equiv(D)$ in $Q_{k, \tau}$. Since by assumption there holds $u_{0 r} \leq v_{0 r}$ a.e. in $I_{k}$, 6.16) follows from Proposition 6.2.

(b) $\hat{c}_{k-1} \hat{d}_{k-1}>0, \hat{c}_{k} \hat{d}_{k} \leq 0$. We consider two subcases:

$\left(b_{1}\right) \hat{c}_{k}<0, \hat{d}_{k} \geq 0$. In this case $u_{r}$ solves problem $\left(D_{ \pm}^{-}\right)$in $Q_{k, \tau}$, depending on $\pm \hat{c}_{k-1}>0$. Since in both cases $\hat{d}_{k}>0$ or $\hat{d}_{k}=0$ it can be easily checked that $v_{r}$ is an entropy supersolution of problem $\left(D_{ \pm}^{-}\right)$in $Q_{k, \tau}$, depending on $\pm \hat{c}_{k-1}>0$ (see Definition [6.2(ii) and (iii)), hence (6.16) follows from Theorem 6.1

$\left(b_{2}\right) \hat{c}_{k} \leq 0, \hat{d}_{k}>0$. In this case $v_{r}$ solves problem $\left(D_{ \pm}^{+}\right)$in $Q_{k, \tau}$, depending on $\pm \hat{c}_{k-1}>0$. In both cases $\hat{c}_{k}<0$ or $\hat{c}_{k}=0$, we get that $u_{r}$ is an entropy subsolution of problem $\left(D_{ \pm}^{+}\right)$in $Q_{k, \tau}$, depending on $\pm \hat{c}_{k-1}>0$ (see Definition 6.1 $(i)$ and $(i v)$ ), and (6.16) follows from Theorem 6.1

(c) $\hat{c}_{k-1} \hat{d}_{k-1} \leq 0, \hat{c}_{k} \hat{d}_{k}>0$. This case is analogous to $(b)$; we omit the details. 
(d) $\hat{c}_{k-1}<0, \hat{d}_{k-1}=0, \hat{c}_{k}=0, \hat{d}_{k}>0$. It is easily checked that $u_{r}$ is an entropy subsolution and $v_{r}$ is an entropy supersolution of problem $\left(D_{-}^{+}\right)$in $Q_{k, \tau}$ (see Definitions 6.1 $(i v)$ and 6.2 $(i v))$. Again (6.16) follows from Theorem 6.1

(e) $\hat{c}_{k-1}=0, \hat{d}_{k-1}>0, \hat{c}_{k}<0, \hat{d}_{k}=0$. This case is analogous to $(d)$.

\section{Waiting time for global solutions of $(H J)$ and $(C L)$ : Proofs}

In this section we prove the results about the waiting times listed in Section 4.3 We observe that Theorem 4.4 is an immediate consequence of (3.31).

Proof of Theorem 4.5. We only adress the case that $J_{0}\left(x_{j}\right)>0$. Observe that until the waiting time $\tau_{j} \in(0,+\infty]$, the jump discontinuity at $x_{j}$ has a barrier effect in the following sense: by [6, Lemma 5.2], $U_{1}=U\left\llcorner\left(\left(x_{j}, \infty\right) \times\left(0, \tau_{j}\right)\right)\right.$ and $U_{2}=U\left\llcorner\left(\left(-\infty, x_{j}\right) \times\left(0, \tau_{j}\right)\right)\right.$ are the viscosity solutions of the problems

$$
\begin{cases}U_{1 t}+H\left(U_{1 x}\right)=0 & \text { in }\left(x_{j}, \infty\right) \times\left(0, \tau_{j}\right) \\ U_{1 x}=\infty & \text { in }\left\{x_{j}\right\} \times\left(0, \tau_{j}\right) \\ U_{1}=U_{0}\left\llcorner\left(x_{j}, \infty\right)\right. & \text { in }\left(x_{j}, \infty\right) \times\{0\}\end{cases}
$$

and

$$
\begin{cases}U_{2 t}+H\left(U_{2 x}\right)=0 & \text { in }\left(-\infty, x_{j}\right) \times\left(0, \tau_{j}\right) \\ U_{2 x}=\infty & \text { in }\left\{x_{j}\right\} \times\left(0, \tau_{j}\right) \\ U_{1}=U_{0}\left\llcorner\left(-\infty, x_{j}\right)\right. & \text { in }\left(-\infty, x_{j}\right) \times\{0\} .\end{cases}
$$

In view of assumption $\left(H_{4}\right)-(i)$, we consider the case that for all $M>0$ there exists $k_{M}>M$ such that $H\left(k_{M}\right)>H^{+}$(if $H\left(k_{M}\right)<H^{+}$the proof is similar). By $\left(A_{1}\right)$ we have that $\left|U_{0}(x)\right| \leq A_{j}+B\left|x-x_{j}\right|$, where $\left.A_{j}=A+B\left|x_{j}\right|\right)$. We set, for all $k>B$ such that $H(k)>H^{+}$,

$$
v(x, t):=C_{k}+k\left(x-x_{j}\right)-H(k) t \quad \text { for }(x, t) \in\left(x_{j}, \infty\right) \times\left(0, \tau_{j}\right),
$$

where $C_{k}$ is chosen such that

$$
v(x, 0) \geq A_{j}+B\left(x-x_{j}\right) \geq\left(U_{0}\right)^{*}(x) \text { for all } x \geq x_{j} .
$$

By (3.21) and the envelope properties we have that $\left(U_{0}\right)^{*}(x)=U^{*}(x, 0) \geq U_{1}^{*}(x, 0)$ for all $x \geq x_{j}$, thus inequality (7.3) gives

$$
v(x, 0) \geq U_{1}^{*}(x, 0) \text { for all } x \geq x_{j} .
$$

Since $v$ is a viscosity supersolution of (7.1) (see [6, Definition 3.2]), by the comparison principle in [6, Theorem 3.1] and (7.4) we get

$$
\left(U_{1}\right)^{*}(x, t) \leq v(x, t) \text { for all }(x, t) \in\left[x_{j}, \infty\right) \times\left[0, \tau_{j}\right) .
$$

Next, observe that Theorem 3.5 (a) ensures that $U_{1}^{*}(x, t)=U(x, t)$ for all $x>x_{j}$ sufficiently close to $x_{j}$; here, as in Remark 3.1, we have identified $U$ with its continuous representative $\tilde{U}_{j+1}$ in the rectangle $Q_{j+1}=\left(x_{j}, x_{j+1}\right) \times\left(0, \tau_{j}\right)$. Therefore taking the limit as $x \rightarrow x_{j}^{+}$in (7.5) gives

$$
U\left(x_{j}^{+}, t\right) \leq C_{k}-H(k) t \quad \text { for any } t \in\left(0, \tau_{j}\right) .
$$

For all $t$ as above there also holds

$$
U\left(x_{j}^{-}, t\right) \geq U_{0}\left(x_{j}^{-}\right)-H^{+} t
$$


(see inequalities (5.21) in [6] for details). Then from (7.6)-(7.7) we obtain

$$
\left(H(k)-H^{+}\right) t \leq \underbrace{U\left(x_{j}^{-}, t\right)-U\left(x_{j}^{+}, t\right)}_{<0 \text { by }(3.30)}+C_{k}-U_{0}\left(x_{j}^{-}\right) \text {for any } t \in\left(0, \tau_{j}\right) \text {. }
$$

Therefore, letting $t \rightarrow \tau_{j}^{-}$, the claim follows from the estimate $\tau_{j} \leq \frac{C_{k}-U_{0}\left(x_{j}^{-}\right)}{H(k)-H^{+}}$.

Proof of Corollary 4.6. We first prove (4.6). For every $x \in \mathbb{R}$, set $U_{0}(x)=u_{0}([0, x])$, and let $U$ be the global viscosity solution of $(H J)$ with initial datum $U_{0}$. Since $U_{0}$ satisfies assumption $\left(H_{3}\right)$, we can apply the correspondence between $u$ and $U$ stated in Theorem 4.1. Then (4.6) follows from (4.2) and the identifications in (4.3)-(4.4).

It remains to prove that the waiting time is finite if $\left(A_{2}\right)$ is satisfied. Observe that $U_{0}(x)=u_{0}([0, x])(x \in \mathbb{R})$ satisfies $\left(H_{3}\right)$ and $\left(A_{1}\right)$, as $\left\|u_{0 s}\right\|_{\mathcal{M}(\mathbb{R})} \leq C\left(\right.$ see $\left.\left(H_{2}\right)\right)$ and $u_{0 r}$ satisfies $\left(A_{2}\right)$. Applying Theorem 4.5 to the global viscosity solution $U$ of $(H J)$ with initial datum $U_{0}$, the desired results follow from (4.3)-(4.4).

It remains to prove Theorem 4.7, which immediately implies Corollary 4.8. In the proof we distinguish the two different hypotheses, $\left(H_{5}\right)$ and $\left(H_{6}\right)$.

Proof of Theorem 4.7: the case of hypothesis $\left(H_{5}\right)$. We only address the case that $c_{j}>0$ and $\left(H_{5}\right)-(i)$ is satisfied (when $c_{j}<0$ and $\left(H_{5}\right)-(i i)$ holds the proof is similar). Let $\left\{k_{n}\right\}$ be a sequence diverging to $\infty$ such that

$$
\lim _{n \rightarrow \infty} \frac{\left|H\left(k_{n}\right)-H^{+}\right|}{M_{k_{n}}}=\limsup _{k \rightarrow \infty} \frac{\left|H(k)-H^{+}\right|}{M_{k}} \geq C_{0}^{+}>0 .
$$

Since $M_{k}=\left\|H^{\prime}\right\|_{L^{\infty}(k, \infty)} \rightarrow 0$ as $k \rightarrow \infty$, we have that

$$
\lim _{n \rightarrow \infty} M_{k_{n}}=0,
$$

whereas by assumption $\left(H_{4}\right)-(i)$, possibly up to a subsequence (not relabeled), there holds either $H\left(k_{n}\right)>H^{+}$or $H\left(k_{n}\right)<H^{+}$for every $n$. Without loss of generality, we may assume that $H\left(k_{n}\right)>H^{+}$for all $n$.

Let $\operatorname{supp} u_{0 s}^{+} \equiv\left\{x_{1}, \ldots, x_{q}\right\}\left(x_{1}<x_{2}<\cdots<x_{q}\right)$. Below we prove that the waiting time $t_{q}$ associated to $x_{q}$ is finite. By a recursive argument, it follows that all Dirac masses of $u_{0 s}^{+}$disappear in finite time.

Arguing by contradiction, we suppose that $t_{q}=\infty$. Let $T>0$ be fixed arbitrarily. Arguing as in the proof of Proposition 6.2 (ii) (in particular, see (6.11a)), for every $k>0$ and $\zeta \in C^{1}\left([0, T] ; C_{c}^{1}\left(\left[x_{q}, \infty\right)\right), \zeta \geq 0, \zeta(\cdot, T)=0\right.$, we get

$$
\begin{array}{r}
\int_{0}^{T} \int_{x_{q}}^{\infty}\left\{\left[u_{q}-k\right]_{+} \zeta_{t}+\operatorname{sgn}_{+}\left(u_{q}-k\right)\left[H\left(u_{q}\right)-H(k)\right] \zeta_{x}\right\} d x d t \geq \\
\geq-\int_{\mathbb{R}}\left[u_{0 r}-k\right]_{+} \zeta(x, 0) d x-\int_{0}^{T}\left[f_{x_{q}^{+}}-H(k)\right] \zeta\left(x_{q}, t\right) d t .
\end{array}
$$

Let $\gamma>x_{q}$ be arbitrarily fixed. For every $k>0$ and $p \in \mathbb{N}$ large enough we set

$$
\begin{gathered}
\beta_{p}(t):=\chi_{[0, T-1 / p]}(t)+p(T-t) \chi_{(T-1 / p, T](t)} \quad(t \in(0, T)) \\
\zeta_{k, p}(x, t)= \begin{cases}1 & \text { if } x_{q} \leq x \leq \gamma+M_{k}(T-t)-\frac{1}{p}, \\
p\left[\gamma+M_{k}(T-t)-x\right] & \text { if } \gamma+M_{k}(T-t)-\frac{1}{p}<x<\gamma+M_{k}(T-t), \\
0 & \text { if } x \geq \gamma+M_{k}(T-t)\end{cases}
\end{gathered}
$$


for $(x, t) \in \mathbb{R} \times(0, T)$. One easily sees that, by the definitions of $M_{k}$ and $\zeta_{k, p}$,

$$
\int_{0}^{T} \int_{x_{q}}^{\infty} \underbrace{\left\{\left[u_{q}-k\right]_{+} \partial_{t} \zeta_{k, p}+\operatorname{sgn}_{+}\left(u_{q}-k\right)\left[H\left(u_{q}\right)-H(k)\right] \partial_{x} \zeta_{k, p}\right\}}_{\leq 0} \beta_{p}(t) d x d t \leq 0 .
$$

Choosing $\zeta(x, t)=\zeta_{k, p}(x, t) \beta_{p}(t)$ in (17.10) and letting $p \rightarrow \infty$, this implies that

$$
\int_{0}^{T}\left[f_{x_{q}^{+}}(t)-H(k)\right] d t+\int_{x_{q}}^{\gamma+M_{k} T}\left[u_{0 r}-k\right]_{+} d x \geq \int_{x_{q}}^{\gamma}\left[u_{q}(x, T)-k\right]_{+} d x \geq 0,
$$

whence, by the second inequality in (3.14),

$$
\begin{gathered}
\int_{0}^{T}\left[f_{x_{q}^{+}}(t)-f_{x_{q}^{-}}(t)\right] d t+\int_{x_{q}}^{\gamma+M_{k} T}\left[u_{0 r}-k\right]_{+} d x \geq \\
\geq \int_{0}^{T}\left[H(k)-f_{x_{q}^{-}}(t)\right] d t \geq\left[H(k)-H^{+}\right] T .
\end{gathered}
$$

Since $t_{q}=\infty$, it follows from (3.16)-(3.17) that

$$
\int_{0}^{T}\left[f_{x_{q}^{+}}(t)-f_{x_{q}^{-}}(t)\right] d t \leq u_{0 s}^{+}\left(\left\{x_{q}\right\}\right) \quad \text { for all } T>0 .
$$

Let $\left\{k_{n}\right\}$ be any sequence satisfying (7.8)-(7.9) and $H\left(k_{n}\right)>H^{+}$for all $n$. From (7.11)-(7.12) (written with $k=k_{n}$ ), for every $T>0$ and $\gamma>x_{q}$ we get

$$
\left[H\left(k_{n}\right)-H^{+}\right] T \leq u_{0 s}^{+}\left(\left\{x_{q}\right\}\right)+\int_{x_{q}}^{\gamma+M_{k_{n}} T}\left[u_{0 r}-k_{n}\right]_{+} d x .
$$

Set $T_{n}:=\frac{2 u_{0 s}^{+}\left(\left\{x_{q}\right\}\right)}{C_{0}^{+} M_{k_{n}}}$. Then from (7.8) we obtain

$$
\lim _{n \rightarrow \infty}\left[H\left(k_{n}\right)-H^{+}\right] T_{n}=\lim _{n \rightarrow \infty} \frac{2 u_{0 s}^{+}\left(\left\{x_{q}\right\}\right)\left|H\left(k_{n}\right)-H^{+}\right|}{C_{0}^{+} M_{k_{n}}} \geq 2 u_{0 s}^{+}\left(\left\{x_{q}\right\}\right) .
$$

Moreover, there holds

$$
\lim _{n \rightarrow \infty} \int_{x_{q}}^{\gamma+M_{k_{n}} T_{n}}\left[u_{0 r}-k_{n}\right]_{+} d x=0,
$$

since $\gamma+M_{k_{n}} T_{n}=\gamma+2 u_{0 s}^{+}\left(\left\{x_{q}\right\}\right) / C_{0}^{+}$and $u_{0 r} \in L_{\text {loc }}^{1}(\mathbb{R})$. By (7.14)-(7.15), choosing $T=T_{n}$ in (7.13) and letting $n \rightarrow \infty$ we obtain $u_{0 s}^{+}\left(\left\{x_{q}\right\}\right) \leq 0$, a contradiction.

Proof of Theorem 4.7: the case of hypothesis $\left(H_{6}\right)$. Let $\left(H_{6}\right)-(i)$ be satisfied and

$$
H(k)<H^{+} \text {for } k \geq \bar{k} \quad(\bar{k}>0)
$$

(in case of $\left(H_{6}\right)$-(ii) the proof is similar). Fix $x_{j} \in \operatorname{supp} u_{0 s}^{+}$and let $w \in C\left([0, \infty) ; \mathcal{M}^{+}(\mathbb{R})\right)$ be the global entropy solution of problem $(C L)$ with initial data

$$
w_{0}:=\max \left\{u_{0 r}, \bar{k}\right\}+u_{0 s}^{+},
$$

satisfying the compatibility conditions in $\operatorname{supp} w_{0 s}=\operatorname{supp} u_{0 s}^{+}=\left\{x_{1}, \ldots, x_{q}\right\}$. By the comparison principle (see Theorem 4.2), it suffices to prove that the waiting time $\tilde{t}_{j}$ associated to each $x_{j}(j=1, \ldots, q)$ is finite.

Since $w_{0 r} \geq \bar{k}$ a.e. in $\mathbb{R}$ and $w_{0 s} \geq 0$ in $\mathcal{M}(\mathbb{R})$, it follows from (3.4), using a proper sequence of test functions, that $w_{r} \geq \bar{k}$ a.e. in $S$. Hence $w$ also is the global entropy solution of the Cauchy problem

$$
\begin{cases}w_{t}+[\tilde{H}(w)]_{x}=0 & \text { in } S=\mathbb{R} \times \mathbb{R}^{+} \\ w=w_{0} & \text { in } \mathbb{R} \times\{0\}\end{cases}
$$


where $\tilde{H}(w):=H\left((w-\bar{k})^{+}+\bar{k}\right)$, satisfying the compatibility conditions at every $x_{j} \in \operatorname{supp} w_{0 s}=\operatorname{supp} u_{0 s}^{+}$. By the definition of $\tilde{H}$ and assumption (7.16), there holds

$$
\lim _{u \rightarrow \infty} \tilde{H}(u)=\sup _{u \in \mathbb{R}} \tilde{H}(u)=H^{+} .
$$

For every $j=1, \ldots, q$ let $h_{x_{j}^{ \pm}} \in L_{\text {loc }}^{\infty}(0, \infty)$ be the functions relative to $w$ given by Proposition 3.3 Then by (3.12) and (7.17) we get

$$
h_{x_{j}^{+}}(t)=H^{+} \quad \text { for a.e. } t \in\left(0, t_{j}\right) \text {. }
$$

By contradiction, let $\tilde{t}_{j}=\infty$. Then by (3.16) and (7.18) we get

$$
\int_{0}^{\infty}\left[H^{+}-h_{x_{j}^{-}}(t)\right] d t \leq c_{j} .
$$

Fix any $\gamma<x_{j}$ such that $u_{0 s}^{+}\left\llcorner I=0\right.$, where $I \equiv\left(\gamma, x_{j}\right)$. Consider the singular Cauchy-Dirichlet problem

$$
\begin{cases}v_{t}+[\tilde{H}(v)]_{x}=0 & \text { in } I \times(0, \infty) \\ v=\infty & \text { in }\left\{\gamma, x_{j}\right\} \times(0, \infty) \\ v=w_{0 r} & \text { in } I \times\{0\} .\end{cases}
$$

By Definition $6.1(i)$ the restriction $w\llcorner(I \times(0, \infty))$ is a subsolution of (7.20), whereas by Theorem $3.2(i)$ there exists a unique global entropy solution $v \in C\left([0, \infty) ; L^{1}(I)\right)$, $v \geq 0$ of (7.20). Then by Theorem 6.1 we get

$$
w \leq v \quad \text { a.e. in } I \times(0, \infty) .
$$

Let $g_{x_{j}^{-}}, g_{\gamma^{+}} \in L_{\mathrm{loc}}^{\infty}(0, \infty)$ be the functions relative to $v$ given by Proposition 5.4 Arguing as for (7.18), from (5.31a) we get

$$
g_{\gamma^{+}}(t)=H^{+} \geq g_{x_{j}^{-}}(t) \text { for a.e. } t>0 \text {. }
$$

On the other hand, in view of (7.21), arguing as in the proof of Proposition 6.2( $($ ii) gives

$$
h_{x_{j}^{-}}(t) \leq g_{x_{j}^{-}}(t) \text { for a.e. } t>0,
$$

whence by inequality (7.19)

$$
\int_{0}^{\infty}\left[H^{+}-g_{x_{j}^{-}}(t)\right] d t \leq c_{j}
$$

Fix any $T>0$. From the weak formulation (3.2), by a standard argument we get

$$
\int_{I} v(x, T) \rho(x) d x=\int_{I} w_{0 r}(x) \rho(x) d x+\iint_{I \times(0, T)} \tilde{H}(v(x, t)) \rho^{\prime}(x) d x d t
$$

for every $\rho \in C_{c}^{1}(I)$. By a proper choice of $\rho=\rho_{n} \rightarrow \chi_{I}$ as $n \rightarrow \infty$, we get

$$
\|v(\cdot, T)\|_{L^{1}(I)}=\int_{I} w_{0 r}(x) d x+\int_{0}^{T}\left[H^{+}-g_{x_{j}^{-}}(t)\right] d t \leq\left\|w_{0 r}\right\|_{L^{1}(I)}+c_{j}=: D_{0} ;
$$

here we have used that for all $\beta \in C_{c}(0, \infty)$ (see (5.29)-(5.30) ) there holds

$$
\begin{aligned}
& \lim _{x \rightarrow x_{j}^{-}} \int_{0}^{\infty} \tilde{H}(v(x, t)) \beta(t) d t=\int_{0}^{\infty} g_{x_{j}^{-}}(t) \beta(t) d t, \\
& \lim _{x \rightarrow \gamma^{+}} \int_{0}^{\infty} \tilde{H}(v(x, t)) \beta(t) d t=\int_{0}^{\infty} g_{\gamma^{+}}(t) \beta(t) d t,
\end{aligned}
$$

and (7.22)-(7.23). 
Similarly, for a.e. $y \in\left(\gamma, x_{j}\right)$, a suitable choice of $\rho=\rho_{n} \rightarrow \chi_{(\gamma, y)}$ in (7.24) implies

$$
\int_{\gamma}^{y} v(x, T) d x=\int_{\gamma}^{y} w_{0 r}(x) d x+\int_{0}^{T}\left[H^{+}-\tilde{H}(v(y, t))\right] d t,
$$

whence, by integration with respect to $y$ and (7.25),

$$
\int_{0}^{T}\left(\int_{I}\left[H^{+}-\tilde{H}(v(y, t))\right] d y\right) d t \leq \int_{I}\left(\int_{\gamma}^{y} v(x, T) d x\right) d y \leq D_{0}|I| .
$$

By (7.17), this implies that

$$
\int_{0}^{T}\left\|\tilde{H}(v(\cdot, t))-H^{+}\right\|_{L^{1}(I)} d t \leq D_{0}|I| .
$$

By the arbitrariness of $T$, there exists a sequence $T_{k} \rightarrow \infty$ such that

$$
\left\|\tilde{H}\left(v\left(\cdot, T_{k}\right)\right)-H^{+}\right\|_{L^{1}(I)} \rightarrow 0,
$$

whence (possibly up to a subsequence, not relabeled)

$$
\tilde{H}\left(v\left(x, T_{k}\right)\right) \rightarrow H^{+} \text {for a.e. } x \in I .
$$

In view of (7.17), this implies that

$$
v\left(x, T_{k}\right) \rightarrow \infty \text { for a.e. } x \in I,
$$

whence $\left\|v\left(\cdot, T_{k}\right)\right\|_{L^{1}(I)} \rightarrow \infty$. However, this contradicts estimate (7.25).

Acknowledgements. MB acknowledges the MIUR Excellence Department Project awarded to the Department of Mathematics, University of Rome Tor Vergata, CUP E83C18000100006, as well as the grant of the University of Rome Tor Vergata "Mission: sustainability - Formation and evolution of singularities".

\section{REFERENCES}

[1] C. Bardos, A. Y. Le Roux \& J. C. Nedelec, First order quasilinear equations with boundary condition, Comm. Partial Differential Equations 4 (1979), 1017-1034.

[2] G. Barles, An introduction to the theory of viscosity solutions for first-order HamiltonJacobi equations and applications, in: "Hamilton-Jacobi equations: approximations, numerical analysis and applications", pp. 49-109, Lecture Notes in Math. 2074, Fond. CIME/CIME Found. Subser. (Springer, Heidelberg, 2013).

[3] M. Bertsch, F. Smarrazzo, A. Terracina \& A. Tesei, Radon measure-valued solutions of first order hyperbolic conservation laws, Adv. in Nonlinear Anal. 9 (2020), 65-107.

[4] M. Bertsch, F. Smarrazzo, A. Terracina \& A. Tesei, A uniqueness criterion for measurevalued solutions of scalar hyperbolic conservation laws, Atti Accad. Naz. Lincei Rend. Lincei Mat. Appl. 30 (2019), 137-168.

[5] M. Bertsch, F. Smarrazzo, A. Terracina \& A. Tesei, Signed Radon measure-valued solutions of flux saturated scalar conservation laws, Discrete \& Continuous Dynamical Systems - A 40(6) (2020) 3143-3169.

[6] M. Bertsch, F. Smarrazzo, A. Terracina \& A. Tesei, Discontinuous viscosity solutions of first order Hamilton-Jacobi equations, preprint (2020), arXiv:1906.05625v2.

[7] V. Caselles, Scalar conservation laws and Hamilton-Jacobi equations in one-space variable, Nonlinear Anal. 18 (1992), 461-469.

[8] M. G. Crandall, L. C. Evans \& P. L. Lions, Some properties of viscosity solutions of Hamilton-Jacobi equations, Trans. Amer. Math. Soc. 282 (1984), 487-502.

[9] M.G. Crandall \& P.L. Lions, Viscosity solutions of Hamilton-Jacobi equations, Trans. Amer. Math. Soc. 277 (1983), 1-41.

[10] F. Demengel \& D. Serre, Nonvanishing singular parts of measure valued solutions of scalar hyperbolic equations, Comm. Partial Differential Equations 16 (1991), 221-254.

[11] L. C. Evans, Envelopes and nonconvex Hamilton-Jacobi equations, Calc. Var \& PDE 50 (2014), 257-282. 
[12] L. C. Evans \& R. F. Gariepy, Measure Theory and Fine Properties of Functions (CRC Press, 1992).

[13] A. Friedman, Mathematics in Industrial Problems, Part 8, IMA Volumes in Mathematics and its Applications 83 (Springer, 1997).

[14] H. Ishii, Perron's method for Hamilton-Jacobi equations, Duke Math. J. 55 (1987), 368384 .

[15] K. H. Karlsen \& N. H. Risebro, A note on front tracking and the equivalence between viscosity solutions of Hamilton-Jacobi equations and entropy solutions of scalar conservation laws, Nonlinear Anal. 50 (2002), 455-469.

[16] T.-P. Liu \& M. Pierre, Source-solutions and asymptotic behavior in conservation laws, J. Differential Equations 51 (1984), 419-441.

[17] O. A. Ladyženskaja, V. A. Solonnikov \& N. N. Ural'ceva, Linear and Quasi-Linear Equations of Parabolic Type (Amer. Math. Soc., 1991).

[18] D. S. Ross, Two new moving boundary problems for scalar conservation laws, Comm. Pure Appl. Math 41 (1988), 725-737.

[19] D. S. Ross, Ion etching: An application of the mathematical theory of hyperbolic conservation laws, J. Electrochem. Soc. 135 (1988), 1235-1240.

[20] A. Terracina, Comparison properties for scalar conservation laws with boundary conditions, Nonlinear Anal. 28 (1997), 633-653.

Dipartimento di Matematica, Università di Roma "Tor Vergata", Via della Ricerca Scientifica, 00133 Roma, italy, and Istituto per le Applicazioni del Calcolo "M. PiCONE", CNR, Roma, ITALy

E-mail address: bertsch.michiel@gmail.com

Università Campus Bio-Medico di Roma, Via Alvaro del Portillo 21, 00128 Roma, ITALY

E-mail address: flavia.smarrazzo@gmail.com

Dipartimento di Matematica "G. Castelnuovo", Universitì "Sapienza" di Roma, P.le A. Moro 5, I-00185 Roma, Italy

E-mail address: terracina@mat.uniroma1.it

Dipartimento di Matematica "G. Castelnuovo", Università "Sapienza" di Roma, P.le A. Moro 5, I-00185 Roma, Italy, and Istituto per le Applicazioni del Calcolo "M. PiCONE", CNR, ROMA, ITALY

E-mail address: albertotesei@gmail.com 